\title{
Niveles y subniveles de precariedad extrema en México: una metodología de grupos con condiciones laborales ordenadas
}

\section{Levels and sub-levels of extreme precariousness in Mexico: A methodology of groups with orderly working conditions}

\author{
Miguel Ángel Mendoza-González* \\ Selene Fabiola Cruz-Calderón** \\ Marcos Valdivia-López***
}

\section{Resumen}

Esta investigación se enfoca en los empleos con condiciones laborales de precariedad extrema, que se identifican por estar en la linea de pobreza extrema por sus ingresos precarios y por ser heterogéneos con respecto a cinco condiciones adicionales de precariedad laboral: sin sindicato, jornada precaria, sin prestaciones, sin seguridad social y contrato precario. Con el objetivo de analizar la heterogeneidad se utilizó una metodología de grupos para identificar niveles y subniveles, ordenados numéricamente de menor a mayor precariedad laboral extrema. También se estimaron modelos probit-pool ordenados por niveles y subniveles de precariedad laboral extrema, explicados por un conjunto de variables del mercado laboral y de contexto, con el objetivo de elaborar simulaciones para medir los efectos marginales en los cambios en la probabilidad de ocurrencia, por nivel y subnivel de precariedad laboral extrema. Los resultados más importantes muestran que los empleos con precariedad extrema alcanzaron a 2.6 millones de trabajadores en 2018; su

* Universidad Nacional Autónoma de México, División de Posgrado de la Facultad de Economía. Dirección: Ciudad Universitaria, Edificio de Posgrado de la Facultad de Economía, cubículo 213, C.P. 10450, Coyoacán, Ciudad de México, México. Correo: mendozag@unam. mx ORCID: http://orcid.org/0000-0003-0119-6300

** Universidad Nacional Autónoma de México, División de Posgrado de la Facultad de Economía. Correo: eara_sel@hotmail.com ORCID: http://orcid.org/0000-0003-0119-6300

*** Universidad Nacional Autónoma de México, Centro Regional de Investigaciones Multidisciplinarias. Dirección: Av. Universidad s/n, Circuito 2, 62210, Col. Chamilpa, Cuernavaca, Morelos, México. Correo: marcosv@correo.crim.unam.mx ORCID: http://orcid. org/0000-0003-0119-6300 
comportamiento se vincula con el desempleo en México, y las condiciones de precariedad laboral extrema con mayor probabilidad de ocurrencia sucedieron con ingresos precarios extremos, contrato y jornada precaria, y sin seguridad social.

Palabras clave: nivel y subnivel de precariedad laboral extrema; mercado laboral; modelo probit-pool ordenado; México.

Clasificación JEL: C38, J01, J21, J44, R23.

\begin{abstract}
This research focuses on jobs with extremely precarious working conditions, identified as being on the extreme poverty line due to their (precarious) income and being heterogeneous with respect to five additional precarious working conditions: no union, a precarious working day, no benefits or social security and precarious contracts. In order to analyze the heterogeneity, a group methodology was used to identify levels and sub-levels, numerically arranged from least to greatest extreme labor precariousness. Pooled probit models were also estimated, arranged by levels and sub-levels of extreme labor precariousness, explained by a set of labor market and context variables, with the aim of developing simulations to measure the marginal effects on changes in the likelihood of occurrence, by level and sub-level of extreme job precariousness. The most important results show that in 2018, 2.6 million workers had extreme job precariousness. Their behavior is linked to unemployment in Mexico, while extreme job precariousness with the highest probability of occurrence was found when conditions of extremely precarious income, contracts and working hours, with no social security, existed.
\end{abstract}

Keywords: level and sub-level of extreme job insecurity; working market; ordered pooled Probit model; Mexico.

JEL classification: C38, J01, J21, J44, R23.

\title{
1. Introducción
}

Las nuevas formas de trabajo, originadas desde los ochenta y fortalecidas en los noventa en el marco de la globalización, se han caracterizado por la combinación de la reestructuración de los procesos productivos y la flexibilización del trabajo. En esta línea, diversas investigaciones se han enfocado en identificar los elementos para analizar las transformaciones que distinguen el nuevo ciclo social y el papel del trabajo en las configuraciones sociales emergentes (Guadarrama, Hualde y López, 2012). Las relaciones laborales han sufrido intensos procesos de redefinición por la vía formal, a través del cambio de las leyes laborales o por el surgimiento de nuevas prácticas de empleo. Al respecto, la OIT (2012) y Weller (2017) han señalado a la creciente precarización del empleo como uno de 
los rasgos más sobresalientes de la dinámica de los mercados laborales latinoamericanos.

La precariedad laboral originalmente fue identificada como la inestabilidad en el puesto de trabajo, ya sea por la inexistencia de contrato o por contratos por tiempo determinado (Barattini, 2009). Pero, a juicio de Mora (2005), el fenómeno tiene un carácter multidimensional, por lo menos en cuatro aspectos: 1) el vínculo entre procesos de precarización, desregulación de los mercados de trabajo y reorganización productiva; 2) el cambio en los modelos de regulación laboral, la relación entre los modelos de relación laboral y la ciudadanía social; 3) la pérdida de seguridad en la continuidad del empleo; y 4) la vulnerabilidad social. Por ello, el concepto de precariedad laboral refleja aspectos del deterioro de las condiciones laborales a partir de la reestructuración productiva, como son la existencia de empleos con jornadas laborales superiores a las de la ley, con ausencia de prestaciones, carencia de organización sindical, falta de contrato legal y de seguridad social, empleos temporales o eventuales, no calificados y fuera de contrataciones colectivas (Oliveira, 2006; Mora, 2005, 2012; Román, 2013; Quiñones y Rodríguez, 2015) . En México, el estudio de las condiciones laborales de los asalariados se convirtió en un fenómeno relevante, no por la falta absoluta de puestos de trabajo, sino por el fomento a la contratación de empleos con condiciones laborales no adecuadas, bajo un régimen de organización del trabajo y de integración profesional basado en la inseguridad social (García, 2010; Guadarrama, Hualdey y López, 2012). La situación laboral en México se ha caracterizado por escenarios cambiantes; en los noventa la industria mexicana mantuvo alguna capacidad de absorción de mano de obra, en gran parte por la expansión de las empresas maquiladoras. Pero, a inicios de la década de 2000, la salida de muchas de estas empresas, por la reducción de la demanda norteamericana hacia las exportaciones mexicanas, permitió que el comercio y los servicios absorbieran gran parte del crecimiento de la fuerza de trabajo mexicana en condiciones poco favorables (García, 2009).

En este contexto, nuestra investigación aporta un enfoque nuevo en la discusión de la precariedad laboral en México. En primer lugar, la población objetivo de la investigación es la del empleo precario extremo, que se caracteriza por las peores condiciones laborales y por obtener ingresos por su trabajo iguales a la línea de pobreza extrema urbana establecida por el Consejo Nacional de Evaluación de la Política Social (Coneval). Estos ingresos laborales no cubren las necesidades básicas y, por lo regular, se combinan con condiciones laborales sin las prestaciones a las que se debería tener derecho, sin seguridad social, con jornadas fuera de la Ley Federal del Trabajo, contratos temporales o ausencia de ellos, y sin afiliación sindical. 
Este condicionamiento de la precariedad laboral extrema abarca formas de inserción y relaciones laborales muy heterogéneas que, ante la dificultad de su comparabilidad y homologación en una sola categoría, nos permiten hablar de niveles y subniveles, que en la literatura se identifican por las dimensiones de precariedad presentes en diversos grados y modalidades en todas las formas de empleo, las cuales contribuyen a la incertidumbre, vulnerabilidad y dependencia de los trabajadores (Cano, 1998, 2004; Rubio, 2010).

La segunda aportación es la aplicación de una metodología de grupos (Olsthoorm, 2014; Spanos, 2003; Shao, 2003; Kerns, 2011) para identificar niveles y subniveles, ordenados numéricamente de menor a mayor precariedad laboral extrema. Con esta metodología se construyeron combinaciones de acuerdo con la teoría de conjuntos con seis condiciones de precariedad laboral extrema (ingreso de precariedad extrema, sin sindicato, jornada precaria, sin prestaciones, sin seguridad social y contrato precario) para seis niveles $(0,1, . ., 5)$ y treinta subniveles de precariedad laboral extrema $(0,1, \ldots, 29)$.

En tercer lugar, se elaboran simulaciones con base en modelos probit-pool para medir los efectos marginales en los cambios de un conjunto de variables del mercado laboral y de contexto, en la probabilidad de ocurrencia por nivel y subnivel de precariedad laboral extrema en México para el periodo 2005-2018. Los resultados más importantes muestran que el empleo en condiciones de precariedad extrema fue de 2.6 millones de trabajadores en 2018; su comportamiento se vincula con el desempleo en México; y las condiciones de precariedad laboral extrema con mayor probabilidad de ocurrencia se dieron con ingresos precarios, contrato y jornada precaria, y sin seguridad social.

Estos tres planteamientos de discusión sobre la precariedad laboral se desarrollan en los siguientes apartados, junto con esta introducción. En el segundo apartado se hace una revisión sobre la literatura sobre precariedad, relevante para nuestro planteamiento de precariedad laboral extrema. La metodología con grupos y modelos probabilísticos con características ordenadas y condicionadas se desarrolla en el apartado tres. En el cuarto apartado se presentan las especificaciones de los datos, la construcción de indicadores y el análisis de las tendencias del empleo precario extremo, de sus niveles y subniveles. En el apartado quinto se estiman los modelos probit-pool ordenados con las condiciones de precariedad laboral extrema, un conjunto de variables del mercado laboral, socioeconómicas y de contexto, y se presentan los resultados de las simulaciones de los efectos marginales del mercado laboral y socioeconómicas sobre los cambios en las probabilidades en los niveles y subniveles de precariedad laboral con mayor 
ocurrencia para el periodo 2005-2018. En el apartado seis se analizan las conclusiones más importantes de la investigación.

\section{Revisión de la literatura relevante sobre la precariedad}

La evolución de las relaciones laborales y del mercado de trabajo interactúan con la precarización, haciéndola parte de un proceso histórico que involucra la pérdida de bienestar de los trabajadores como individuos, y un sentido colectivo de vulnerabilidad creciente. Diversos estudios muestran coincidencias en las percepciones y vivencias de la precariedad entre los trabajadores, que se resume como una "visión fatalista" de la situación del mercado de trabajo, o bien de naturalización de la precariedad (Cano, 2004). Bajo estas condiciones, la capacidad y autonomía de los trabajadores para planificar y controlar su vida profesional y social se reduce, al mismo tiempo que existe una asimetría de las relaciones de poder que definen la relación laboral (Cano, 1998). En este sentido, los trabajadores con empleo inestable viven la precariedad como un problema de inseguridad e incertidumbre que va más allá del ámbito laboral, puesto que imposibilita la participación en la estructura del consumo (particularmente la adquisición de vivienda) e impide cualquier proyecto de vida a largo plazo (Bilbao, 1999). Así, la inseguridad del empleo genera una visión pesimista, afecta la visión de largo plazo y lleva a preocuparse sólo del presente (Cano, 2004).

Por largo tiempo, el interés de muchas investigaciones se enfocó en los trabajadores por cuenta propia, por sus bajos niveles de ingreso y ausencia de prestaciones sociales. Pero los signos de la precariedad de los trabajadores asalariados mexicanos se han acentuado: en el año 2000 el 55\% de la población ocupada asalariada tenía acceso a instituciones de salud y sólo $54 \%$ tenía contratos escritos (García, 2010). De acuerdo con Salas (2000), en México se ha vuelto común la proliferación de nuevos puestos de trabajo que no son de carácter permanente, y los pagos por honorarios se han convertido en la única forma de contratación para gran cantidad de trabajadores. Esta creciente inestabilidad provoca la tendencia a la fragmentación de las jornadas laborales con prevalencia de trabajos a tiempo parcial y donde no es difícil encontrar jornadas superiores a las 60 horas. Además, la cobertura de prestaciones sociales tiende a disminuir, lo que refleja la tendencia de la calidad media de los empleos asalariados al deterioro.

La población joven y la de menor escolaridad carecen en mayor medida de contratos permanentes y prestaciones de salud; en las ramas de la construcción y los servicios diversos se encuentran más este tipo de condiciones. 
Los trabajadores con contratos permanentes y prestaciones de salud están más representados entre los individuos de edad madura, los más escolarizados y entre quienes laboran en los servicios sociales, en el gobierno y en la industria extractiva, actividades económicas usualmente ejercidas bajo condiciones formales de contratación (Castel, 1997; Neffa, Tupac y Pérez, 2000; Neffa, 2000). Entre los asalariados prevalecen los bajos niveles de ingresos, así como la peor situación relativa de quienes no tienen contratos ni prestaciones de salud; los empleos temporales presentan una situación intermedia entre quienes no tienen contratos y los que cuentan con contratos permanentes (García, 2012). Así, con mayor magnitud de la precariedad laboral del trabajador, aparecen los riesgos de rupturas sociales y familiares, menor sociabilidad, pérdida de identidad, mayor sufrimiento psíquico y mental, y en general peores condiciones de calidad de vida (Castel, 1997; Neffa, 2010).

Desde lo económico, los estudios se han enfocado en entender que la reorganización de las relaciones capital-trabajo explican el fenómeno de la precariedad en tres ámbitos: el reparto del riesgo, la distribución del producto y el proceso de trabajo (Recio, 2015). El proceso, que afecta a las personas que experimentan el deterioro de las condiciones laborales, tiene impactos potenciales para el conjunto de asalariados y para la producción de bienes y servicios. García y Oliveria (2001) plantean que la economía mexicana se ha caracterizado por una acentuada heterogeneidad laboral, donde los trabajadores asalariados coexisten con los no asalariados (patrones, por cuenta propia, no remunerados), la gran empresa con los microestablecimientos, los sectores más modernos de los servicios con los vinculados a la economía de subsistencia, los empleos formales con las actividades informales. Una heterogeneidad estructural que se acentúa con la globalización de los procesos productivos y donde la naturaleza de las actividades asalariadas se ve amenazada por el reemplazo de la precariedad de los empleos como rasgo dominante de la organización del trabajo (Castel, 1997; Oliveira, 2006).

En este sentido, Bonilla (2015) establece que la integración de la economía mexicana a los circuitos de producción, comercialización y flujos de financiamiento global, iniciada con la reestructuración de los ochenta, ha sido marcada por un permanente estancamiento económico, al que se le han dado interpretaciones, como el ser resultado de la política de reestructuración económica basada en el modelo neoliberal de liberación comercial y con una alta regencia del mercado; además de la sumisión al ciclo económico estadounidense y a las políticas neoliberales de estabilidad macroeconómica. Para Rojas y Salas (2007) es importante recordar la necesidad de ubicar en el contexto nacional el fenómeno de la precariedad laboral, para 
lo cual destacan las características principales de la estructura del empleo en México, enumerándolas de la siguiente manera: 1) la relativa estabilidad de la proporción del trabajo asalariado en el total del empleo, cuya desaceleración después de la década de los setenta se debió a los obstáculos al crecimiento sostenido de la economía; 2) el reducido volumen de desempleo abierto, que esconde la inserción de los trabajadores en actividades poco redituables y con condiciones de trabajo poco favorables, y la importancia de la migración hacia Estados Unidos; 3) la importancia de las actividades de pequeña escala, que absorben un número considerable de trabajadores, incluidos los que laboran por cuenta propia y proporcionan mercancías baratas a los grupos de menores ingresos, pero funcionan con bajos niveles de capitalización, dificultades para renovar los bienes de capital, ganancias reducidas, distintos niveles de capitalización y rentabilidad, y ocupan fuerza de trabajo con características muy diversas.

\section{Metodología de grupos y modelos probabilísticos con características ordenadas y condicionadas}

De la revisión de la literatura relevante sobre precariedad laboral, se observa que su medición ha sido objeto de múltiples interpretaciones metodológicas a lo largo de las últimas décadas, lo que conlleva a notables diferencias, debido, en parte, a las dificultades para conceptualizar el término, planteándose enfoques alternativos y algunas veces aparentemente contrapuestos, y que contribuyen a la discusión de alternativas. Para el análisis de la precariedad se han implementado diversas estrategias en la construcción de índices con énfasis en sumatorias simples (Gallo, 2003; Rubio, 2010; Castillo, 2001) y los elaborados con técnicas estadísticas más sofisticadas, como el análisis factorial o componentes principales (Oliveira, 2006; Castillo, 2009; Mora, 2005, 2012; Escotia, 2011; Román y Cervantes, 2013).

La medición de las condiciones laborales puede realizarse tanto a nivel agregado (macro) como a nivel individual (micro). En un análisis macro, Rodríguez-Orregia y Silva (2009) proponen la construcción de un índice de condiciones laborales para cada estado de la República Mexicana, que contempla tres dimensiones: condiciones de igualdad por género e ingreso, trabajo cubierto por la seguridad social y premios salariales por educación, con datos de la Encuesta Nacional de Empleo (ENE) de 2004 y con la elaboración del índice mediante la técnica utilizada en la construcción del Índice de Desarrollo Humano (IDH). En el estudio de García (2009), con indicadores de la ENOE en 2006, se elaboró un análisis factorial para dar 
cuenta de las condiciones de los mercados de trabajo urbanos en México en cuanto a la precariedad, el desempleo, los bajos niveles de ingresos, las jornadas parciales, los niveles de sindicalización, la presencia de trabajadores por cuenta propia y de microempresas. Plantea dos índices de situación laboral, para cada ciudad y diferenciados por sexo, a través de seis variables: 1) ocupados en microempresas precarias (en comercio y servicios); 2) en industria (que no tengan razón social o nombre o que aun teniéndolos no cuentan con contratos y prestaciones); 3) con ingresos bajos; 4) jornada parcial involuntaria; 5) ausencia de contratos permanentes y prestaciones; y 6) no estar sindicalizado.

Por su parte, a nivel micro, Román (2013) propone dos fases metodológicas. Con la primera se mide el concepto de empleo precario a partir de siete indicadores: salario mínimo, temporalidad, seguridad social, jornada de trabajo, prestaciones sociales, sindicato y contrato. Construye una variable dicotómica para identificar el empleo precario (uno si lo tiene y cero cuando no) y categoriza el índice de precariedad laboral de la población joven asalariada mediante el método de componentes principales y su estratificación. La segunda consiste en estimar modelos de regresión logística ordinal para analizar el efecto que tiene la edad, el sexo, el nivel de escolaridad, el sector de actividad, el tipo de ocupación y el tamaño del establecimiento sobre los niveles de precariedad laboral. Con un planteamiento semejante, se encuentra el estudio de Oliveira (2006), para la Encuesta Nacional de Juventud de 2005 en México, quien desarrolla un índice de precariedad para los jóvenes asalariados utilizando un análisis factorial, el cual posteriormente se somete al análisis de regresión lineal múltiple para examinar cuáles de las características contextuales, familiares e individuales de los jóvenes permiten explicar por qué algunos de ellos ocupan empleos con mejores o peores condiciones laborales y niveles de ingreso.

Es importante señalar que una de las problemáticas que destaca Mora (2012), en la formulación y aplicación de metodologías que permitan la medición de la precariedad laboral, es el hecho de que la noción de este concepto puede responder a una buena construcción teórica; pero, comúnmente, cuando se le pretende estudiar empíricamente, se dispone de un conjunto limitado de indicadores, principalmente cuando se hacen estudios que involucran la utilización de las encuestas nacionales de empleo, produciéndose un desfase entre la amplitud del concepto y las limitantes empíricas. Por tanto, la concepción teórica de la precariedad laboral necesariamente determinará la forma en la que se realizará su medición.

Las distintas estrategias de medición mostradas anteriormente son un claro ejemplo de que, ante el carácter multidimensional y de distintas inten- 
sidades de la precariedad laboral, el reto en la definición metodológica para su medición es amplio. Esta revisión bibliográfica permite reflexionar sobre el fenómeno de la precariedad laboral y el reto que implica una elaboración metodológica adecuada para su medición. Se trata de plantear una metodología que permita plasmar la definición conceptual del fenómeno a través de un uso adecuado de los indicadores necesarios que lo representen, sus distintos grados, y cuál es la importancia relativa de cada uno de ellos para abordar de manera más amplia las características particulares del deterioro de la calidad del empleo de los asalariados.

Con la metodología de grupos que proponemos (Olsthoorm, 2014; Spanos, 2003; Shao, 2003; Kerns, 2011), se tiene la ventaja de identificar todas las posibles combinaciones entre las condiciones laborales de los trabajadores y, a su vez, clasificarlas en niveles y subniveles, ordenados numéricamente de menor a mayor precariedad laboral extrema. Con este procesamiento se garantiza la medición y análisis de la heterogeneidad de la precariedad laboral extrema.

\subsection{Identificación de grupos con condiciones laborales ordenadas}

En esta investigación se utiliza el enfoque micro al estilo de Mora (2012), Oliveira (2006), Román (2013) y Román y Cervantes (2013) para analizar el comportamiento de la población ocupada con diferentes condiciones laborales de precariedad que, por la combinación de ellas, se clasifican en niveles y subniveles de precariedad laboral extrema. Para poder identificar y clasificar a la población ocupada se utilizaron los conceptos de la teoría de conjuntos (Olsthoorm, 2014; Spanos, 2003; Shao, 2003; Kerns, 2011). En primer lugar, se establece que existe un proceso generador de información $(P G I)$ que define al universo de las personas ocupadas $U_{p o}$; de este universo se aplica la primera condición (ingreso precario extremo) para definir al grupo de población ocupada con precariedad general, como:

$$
U_{\text {pre,g }}=U_{\text {po }} \mid I_{\text {pre,g }}
$$

donde $I_{p r e, g}$ es una función indicador que asigna valores de uno a los individuos que cumplen con la condición de ser población ocupada con precariedad general, y cero cuando son población ocupada sin precariedad general.

El grupo de población ocupada con precariedad extrema por ingreso general $U_{\text {pre,g, }}$, se estratifica por tipo de precariedad particular definida 
por la combinación de un conjunto de condiciones del mercado laboral. En nuestro caso, la población ocupada con condición de precariedad extrema general está definida por ingresos precarios extremos; y para la precariedad particular se analizan cinco condiciones: 1) sin sindicato; 2) con jornada precaria; 3) sin prestaciones; 4) sin seguridad social; y 5) con contrato precario. ${ }^{1}$ En teoría de conjuntos, cada una de estas condiciones nos muestra los resultados de eventos simples, de manera que podemos identificar dentro de la población ocupada, con precariedad extrema general, aquella que tiene una de las cinco condiciones de precariedad individual y ordenarla de una situación de menor a mayor precariedad extrema individual, de acuerdo con el valor numérico asignado a cada condición. De acuerdo con lo anterior, se pueden escribir cinco grupos de personas ocupadas con precariedad extrema general y una particular, como eventos condicionados:

$$
\begin{aligned}
& A=U_{\text {pre }, \mathrm{g}}\left|I_{\text {pre }, 1} ; B=U_{\text {pre }, \mathrm{g}}\right| I_{\text {pre }, 2} ; C=U_{\text {pre }, \mathrm{g}} \mid I_{\text {pre }, 3} \text {; } \\
& D=U_{\text {pre }, \mathrm{g}}\left|I_{\text {pre, } 4} ; \mathrm{y}, E=U_{\text {pre,g }}\right| I_{\text {pre, } 5},
\end{aligned}
$$

donde cada $I_{p r e, i}$ es una función indicador que toma el valor de uno para identificar a los individuos que cumplen con tener una de las $i=1,2,3,4,5$ condiciones definidas.

Con la definición de los eventos simples se puede escribir el conjunto de resultados como $S=\{A, B, C, D, E\}$ y el espacio de eventos trivial como $\mathfrak{\Im}_{0}=\{\varnothing, S\}$, donde en el conjunto vacío (Ø) se localiza la población ocupada con precariedad extrema general, pero sin precariedad laboral extrema particular.

Para definir los subniveles y niveles de precariedad laboral extrema se aplica el concepto del conjunto potencia, que incluye todas las posibilidades para identificar grupos de individuos con precariedad laboral extrema mixta, con combinaciones de condiciones. El conjunto potencia incluye el espacio de eventos trivial $\left(\mathfrak{\Im}_{0}\right)$ y los grupos de individuos con las combinaciones de condiciones. Las combinaciones posibles, comenzando con el conjunto de resultados simples o grupo con una condición de precariedad laboral extrema, se definen como:

Con una condición:

${ }^{1}$ Cada una de las condiciones laborales se especifica con detalle en la parte de la construcción de los indicadores. 


$$
[(A),(B),(C),(D),(E)]
$$

Con dos condiciones:

$$
\left[\begin{array}{l}
(A, B),(A, C),(A, D),(A, E),(B, C), \\
(B, D),(B, E),(C, D),(C, E),(D, E)
\end{array}\right]
$$

Con tres condiciones:

$$
\left[\begin{array}{c}
(A, B, C),(A, B, D),(A, B, E),(A, C, D),(A, C, E), \\
(A, D, E),(B, C, D),(B, C, E),(C, D, E)
\end{array}\right]
$$

Con cuatro condiciones:

$$
[(A, B, C, D),(A, B, D, E),(A, C, D, E),(B, C, D, E)]
$$

Con cinco condiciones:

$$
[(A, B, C, D, E)]
$$

\subsection{Niveles de precariedad laboral extrema}

Para los niveles de precariedad laboral extrema, Nivel $_{j}$ con $j=0,1,2,3,4,5$, se utilizan los conjuntos de combinación de condiciones, sin importar la valorización de la combinación; es igual de precario extremo el individuo con la combinación de condición $A B$, que $A C$ o $A D$. En el nivel de precariedad extrema cero ( Nivel $_{0}$ ) se tiene a los individuos del conjunto vacío Ø; para el nivel de precariedad extrema uno ( Nivel $_{1}$ ), se consideran los individuos que cumplen con tener una condición de precariedad laboral extrema; y así hasta el nivel de precariedad extrema cinco $\left(\mathrm{Nivel}_{5}\right)$, donde los individuos tienen cinco condiciones de precariedad laboral extrema particular. Con la especificación de los niveles de precariedad laboral extrema se establece que el conjunto potencia contiene como primer elemento al nivel de precariedad extrema cero que es igual al conjunto vacío $\left(\right.$ Nivel $\left._{0}=\varnothing\right)$; en segundo lugar, el nivel uno contiene el conjunto de resultados $S$; y así los niveles subsiguientes contienen las combinaciones de los eventos simples: 


$$
P(S)=\left\{\text { Nivel }_{0}, \text { Nivel }_{1}, \text { Nivel }_{2}, \text { Nivel }_{3}, \text { Nivel }_{4}, \text { Nivel }_{5}\right\}
$$

El conjunto potencia contiene todos los posibles resultados de las posibilidades de niveles de precariedad extrema, donde cada nivel es diferente a los otros y están ordenados, de tal manera que podemos garantizar ir del grupo de individuos con nivel de precariedad extrema más bajo hasta el más alto: $\mathrm{Nivel}_{0}>\mathrm{Nivel}_{1}>\mathrm{Nivel}_{2}<\mathrm{Nivel}_{3}>\mathrm{Nivel}_{4}>\mathrm{Nivel}_{5}$.

\subsubsection{Subniveles de precariedad laboral extrema}

Para la conformación de los subniveles de precariedad laboral extrema, Subnivel $_{j, k}$ con $j=0,1,2,3,4,5$ y $k=1, . ., 10$, son importantes tanto la valorización de las condiciones individuales como las de las combinaciones de condiciones en cada nivel de precariedad extrema. Así, el subnivel de precariedad extrema que contiene al conjunto vacío $(j=0)$ solamente tiene un elemento $(k=1)$, que resulta ser el mismo del Nivel cero; esto es, $\left(\right.$ Subnivel $_{0,1}=$ Nivel $\left._{0}\right)$. Para los subniveles con una condición de precariedad, se tienen cinco posibilidades: Subnivel ${ }_{1,1}=A$, ..., Subnivel $_{1,5}=D$; con dos condiciones de precariedad se tienen diez posibles combinaciones que se identifican como Subnivel $_{2,1}, \ldots$, Subnivel $_{2,10}$; con tres condiciones se identificaron nueve elementos, Subnivel ${ }_{3,1}, \ldots$, Subnivel ${ }_{3,9}$; con cuatro condiciones, son cuatro elementos Subnivel S $_{4,1}, \ldots$, Subnivel $_{4,4} ; \mathrm{y}$, finalmente, con cinco condiciones, un solo elemento Subnivel ${ }_{5,1}$. El conjunto potencia tiene en total 30 posibilidades de subniveles de precariedad laboral, que se definen como:

$$
P(S)=\left\{\begin{array}{l}
\text { Subnivel }_{0,1}, \text { Subnivel }_{1,1}, \ldots, \text { Subnivel }_{2,1}, \ldots, \text { Subnivel }_{2,10} \\
\text { Subnivel }_{3,1}, \ldots, \text { Subnivel }_{3,9}, \text { Subnivel }_{4,1}, \ldots, \text { Subnivel }_{4,4}, \text { Subnivel }_{5,1}
\end{array}\right\}
$$

De la misma manera que en el caso de los niveles de precariedad, cada uno de los subniveles de precariedad extrema son diferentes y están ordenados para identificar los subniveles de precariedad desde el más bajo hasta el más alto: Subnivel $_{0,1}>$ Subnivel $_{1,1}>, \ldots,>$ Subnivel $_{1,5}>$ Subnivel $_{5,1}$. 


\subsection{Modelo probabilístico con respuestas condicionadas ordenadas}

En general, nuestra metodología de modelación consiste en explicar los niveles y subniveles de precariedad laboral extrema mediante modelos probabilísticos con respuestas ordenadas (Agresti, 2002; Green, 2003), donde se pueden obtener las probabilidades de ocurrencia asociados con cada nivel y subnivel de precariedad laboral extrema y sus tendencias para el periodo 2005-2018; como explicación de tales probabilidades de ocurrencia de precariedad laboral extrema hay un conjunto de factores del mercado laboral, sociodemográficos y contextuales, tales como escolaridad, edad, sexo, etc., localización urbana o rural de los trabajadores, tamaño de la empresa y sector económico donde laboran.

El modelo probabilístico con respuestas ordenadas que se utiliza en esta investigación se caracteriza por identificar y explicar las condiciones de precariedad en las que se incorporaron los individuos al mercado laboral. En tal sentido, los individuos se ven en la necesidad de aceptar una opción entre un conjunto de $M$ alternativas posibles de trabajos en condiciones de precariedad extrema (Green, 2003; Cabrer, Sancho y Serrano, 2001; García Mora, 2004).

Desde el punto de vista de la modelación econométrica, estas condiciones implican utilizar el enfoque de la variable latente, que supone la existencia de una variable no observable limitada por un rango de opciones en niveles $(0,1, \ldots, 5)$ y subniveles de precariedad laboral extrema $(0,1, \ldots, 29)$, $Y_{i, t}$, que están condicionados a un conjunto de factores explicativos de los individuos apilados por años, $X_{i, t}$. La combinación de $i$ individuos para $t$ años permite compilar un conjunto de indicadores longitudinales tipo pool no balanceado, que permite que $i$ sea diferente en todos los años considerados.

Desde este enfoque, los valores observados de niveles y subniveles de precariedad laboral de los individuos, $Y_{i, t}$, se identifican de acuerdo con el siguiente esquema:

$$
Y_{i, t}\left\{\begin{array}{l}
0 \quad \text { si } Y_{1, t} \geq Y_{i, t}^{*} \\
1 \text { si } Y_{2, t} \geq Y_{i, t}^{*} \geq Y_{1, t} \\
2 \text { si } Y_{3, t} \geq Y_{i, t}^{*} \geq Y_{2, t} \\
\cdots \\
(M-1) \text { si } Y_{i, t}^{*} \geq Y_{t,(M-1)}
\end{array}\right.
$$


donde $\gamma_{1, t}, \gamma_{2, t}, \ldots, \gamma_{t,(M-1)}$, son los valores de los umbrales y barreras para cada año; hay una correspondencia entre el orden de los niveles y subniveles de precariedad laboral y el orden de la precariedad laboral latente; es decir, que si $Y_{i, t}<Y_{j, t}$ implica que $Y_{i, t}^{*}>Y_{j, t}^{*}$.

El modelo de respuestas múltiples ordenadas tipo pool se expresa formalmente a través de la siguiente relación:

donde:

$$
Y_{i, t}^{*}=F\left(X_{i, t} \beta\right)+u_{i, t}
$$

$Y_{i, t}^{*}$ : variable latente (no observada) que cuantifica los distintos niveles y subniveles de precariedad laboral extrema por individuo y por año.

$\mathrm{F}($.): función no lineal de una combinación lineal de los factores explicativos.

$X_{i, t} \beta$ : combinación lineal de las variables por individuo y por año, denominado índice del modelo.

$u_{i, t}$ : son los errores.

La especificación funcional $F($.$) que se propone es el modelo pro-$ bit-pool con respuestas ordenadas (Agresti, 2002; Green, 2003), donde los niveles y subniveles de precariedad laboral extrema latentes de $Y_{i, t}^{*}$ vienen definidos por la siguiente relación:

$$
\begin{aligned}
& \operatorname{Prob}\left(Y_{i, t}=0 \mid x_{i, t}\right)=\Phi\left(-x_{i, t}{ }^{\prime} \beta\right) \\
& \operatorname{Prob}\left(Y_{i, t}=1 \mid x_{i, t}\right)=\Phi\left(\mu_{1}-x_{i, t}{ }_{i, t} \beta\right)-\Phi\left(-x_{i, t}{ }^{\prime} \beta\right) \\
& \operatorname{Prob}\left(Y_{i, t}=2 \mid x_{i, t}\right)=\Phi\left(\mu_{2}-x_{i, t}{ }^{\prime} \beta\right)-\Phi\left(\mu_{1}-x_{i, t}{ }^{\prime} \beta\right) \\
& \operatorname{Prob}\left(Y_{i, t}=J \mid x_{i, t}\right)=1-\Phi\left(\mu_{J-1}-x_{i, t}{ }^{\prime} \beta\right)
\end{aligned}
$$

Para que todas las probabilidades sean positivas, se debe cumplir con la condición: $0<\mu_{1}<\mu_{2}<\ldots<\mu_{J-1}$. El efecto marginal de cada variable exógena sobre la probabilidad de pertenecer a cada categoría se define por:

$$
\begin{aligned}
& \frac{\partial \operatorname{Prob}\left(Y_{i, t}=0 \mid x_{i, t}\right)}{\partial x_{j, t}}=-\Phi\left(x_{i, t}{ }^{\prime} \beta\right) \beta \\
& \frac{\partial \operatorname{Prob}\left(Y_{i, t}=1 \mid x_{i, t}\right)}{\partial x_{j, t}}=\left[\Phi\left(-x_{i, t}{ }^{\prime} \beta\right)-\Phi\left(\mu-x_{i, t}{ }^{\prime} \beta\right)\right] \beta \\
& \frac{\partial \operatorname{Prob}\left(Y_{i, t}=J \mid x_{i, t}\right)}{\partial x_{j, t}}=\Phi\left(\mu_{j}-x_{i, t}{ }^{\prime} \beta\right) \beta
\end{aligned}
$$


Finalmente, los coeficientes estimados en los modelos ordenados no cuantifican directamente el incremento en la probabilidad, dado el aumento unitario en la correspondiente variable independiente. La magnitud de la variación en la probabilidad depende de su nivel original, por tanto, de los valores iniciales de todos y cada una de las variables exógenas y de sus coeficientes (García, 2004). Así, la magnitud del efecto marginal de una variable explicativa $x_{i, t}$ depende del valor del coeficiente $\beta$ y de los valores de la función de densidad $\Phi($.) para cada uno de los individuos. Cuanto más grande sea el valor del coeficiente $\beta$, mayor será el impacto del cambio en el valor del regresor, el cual va a incidir en el valor de la variable latente $Y_{i, t}^{*}$.

\section{Datos, construcción de indicadores y tendencias de precariedad laboral extrema}

\subsection{Datos y construcción de indicadores de precariedad extrema}

Los indicadores para identificar grupos de trabajadores en niveles y subniveles de precariedad extrema se construyeron con el cuestionario ampliado de la Encuesta Nacional de Ocupación y Empleo (ENOE), que publica el INEGI de manera continua para el primer trimestre de 2009 al 2018; en los años 2005 y 2006 se publicó en los dos primeros trimestres y en 2007 y 2008 en el segundo trimestre. Los indicadores utilizados son las condiciones de los individuos que se encuentran en el mercado laboral identificados como población ocupada, entre 15 y 65 años, con ingresos laborales positivos; por lo que en nuestra población objetivo no se considera a la población ocupada no remunerada. En todas las estimaciones de los grupos, como en los modelos probabilísticos, se aplicaron los factores de expansión de cada año para el periodo 2005-2018. Las seis condiciones con las que se identificaron a los trabajadores precarios extremos fueron: 1) ingresos mensuales precarios extremos; 2) no sindicalizados; 3) condiciones del contrato precarios; 4) sin prestaciones; 5) jornada laboral precaria; 6) sin seguridad social.

El ingreso mensual precario extremo se midió de acuerdo con la línea de la pobreza extrema por ingreso (alimentaria más no alimentaria) en zonas urbanas que Coneval reconoce como insuficiente para cubrir las necesidades básicas. En otras investigaciones se considera que el empleo precario se identifica por la inseguridad de los ingresos, que no permiten la reproducción social y son menores a los dos salarios mínimos (Mora, 2012). Con nuestro planteamiento de empleo precario extremo se identificó que el in- 
greso laboral de precariedad extrema fue de 1483 pesos en el mes de marzo de 2018 (véase la Gráfica 1), aumentó en $5.8 \%$ anual de 2005 a 2018, es equivalente al ingreso máximo del séptimo percentil de la distribución del ingreso laboral -sobre todo de 2009 a 2018-y representa una porción menor de los todos los ingresos laborales de referencia que se han analizado en la literatura (Oliveira, 2006; Castillo, 2009; Mora, 2005, 2012; Escotia, 2011; Román y Cervantes, 2013), los cuales se presentan en la Gráfica 1. En promedio, los ingresos laborales extremos son $76 \%$ del ingreso máximo de la población ocupada en el décimo percentil de ingresos que estimamos con la ENOE, $57 \%$ del salario mínimo y 47\% del ingreso de la línea de pobreza urbana definida por Coneval.

\section{Gráfica 1}

Comparación de ingresos laborales. Unidad: pesos

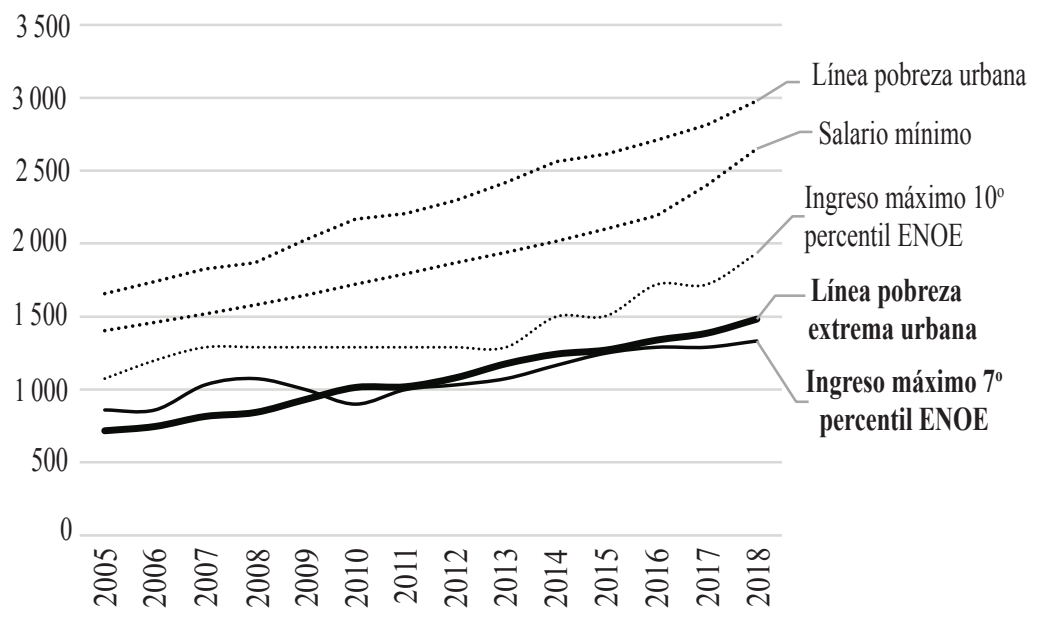

Fuente: Elaboración propia con base en Coneval, Conasami y ENOE, 2005-2018.

El empleo con ingreso laboral de precariedad extrema se estimó en 2.6 millones de personas en 2018, nivel muy parecido a la población ocupada con ingresos laborales máximos definido en el séptimo percentil de la distribución del ingreso medidos con la ENOE, sobre todo después de la crisis económica de 2009 (Gráfica 2). Con respecto a las poblaciones ocupadas compatibles con las otras referencias de ingresos laborales, estimamos que la población ocupada con ingresos máximos en el décimo percentil 
de ingreso representó 1.43 veces de la población ocupada con ingresos con precariedad extrema, la población ocupada con un salario mínimo fue 2.1 veces, y la población ocupada con ingresos en la línea de pobreza urbana 2.7 veces; todos ellos en promedio durante el periodo 2005-2018. Es importante mencionar que el efecto relevante de la crisis económica de 2009 fue duplicar la población ocupada con ingresos laborales en las distintas medidas de salarios insuficientes para cubrir las necesidades de la población, como se presenta en la Gráfica 2.

\section{Gráfica 2}

Población ocupada remunerada por ingresos laborales. Unidad: personas

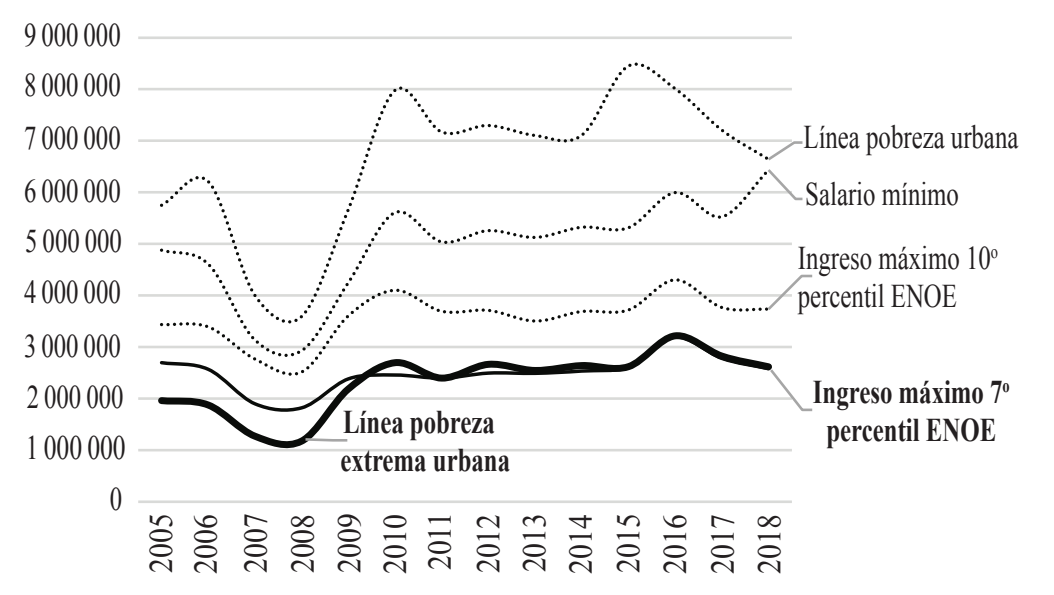

Fuente: Elaboración propia de acuerdo con los límites de ingresos laborales definidos en la Gráfica 1 y aplicados a la población ocupada de la ENOE, 2005-2018.

La segunda condición de precariedad es la de no estar sindicalizado. En la ENOE existe una pregunta específica sobre pertenecer o no a algún sindicato. Desde nuestro punto de vista, el no estar sindicalizado tiene como efecto la pérdida de la posibilidad de presionar sobre mejores condiciones con base en una estructura de apoyo institucional (Quiñones y Rodríguez, 2015). En los distintos estudios sobre precariedad laboral (Barattini, 2009; Bayón, 2006; Mora, 2012), la condición principal del contrato precario se concentró en el empleo temporal, pero con la ENOE se pueden identificar otros aspectos. En nuestra metodología se consideró la población ocupada sin contrato escrito, y en el caso de tenerlo, si es temporal, por obra determinada o de tipo no especificado. 
En el indicador de sin prestaciones, se identificaron los trabajadores sin ninguna de las prestaciones que se consideran en la ENOE: aguinaldo, vacaciones con goce de sueldo y reparto de utilidades.

Otra de las condiciones de precariedad más analizadas es la jornada laboral precaria; para su construcción se utilizó el concepto de jornada completa de la Organización Internacional del Trabajo (OIT), que la define como aquella que se mantiene en el margen de entre 35 a 48 horas semanales. En tal sentido, se definió la jornada laboral precaria tanto por la jornada incompleta, que incluye a los trabajadores con menos de 34 horas, como por la jornada extendida de más de 48 horas (Mora, 2005; 2012).

Y, finalmente, para la condición de trabajadores sin seguridad social se utilizó la falta de acceso a la atención médica de instituciones, que en la ENOE se identifican como: IMSS, Naval, Militar o PEMEX, ISSSTE, ISSSTE estatal, otra institución médica.

Es importante mencionar que revisamos la posibilidad de realizar un análisis de la precariedad para un periodo más amplio, usando la Encuesta Nacional de Empleo Urbano (ENEU) para el periodo 1987-2004, pero con las preguntas de la ENEU sólo se pueden construir cuatro de las seis características de precariedad laboral: ingreso mensual precario, sin prestaciones, jornada laboral precaria y sin seguridad social.

\subsection{Especificación de grupos con niveles y subniveles de precariedad laboral extrema}

Para definir el universo de la población ocupada $\left(U_{p o}\right)$ con precariedad extrema general $\left(U_{\text {pre,g }}\right)$, se construyó la función indicador $I_{p r e, g}$, que toma el valor de 1 si cumple con la condición de obtener un ingreso laboral mensual igual o menor a la línea de pobreza extrema por ingreso en zonas urbanas. Una vez definido el universo de la población ocupada con precariedad extrema general o de ingreso precario extremo, se identificaron los individuos con cada una de las cinco condiciones de precariedad extrema particular, esto es: el grupo de trabadores sin sindicatos $A=U_{\text {pre,g }} \mid I_{\text {pre, } 1}$; el grupo de trabajadores con contrato precario $B=U_{\text {pre, } g} \mid I_{\text {pre }, 2}$; los trabajadores sin prestaciones $C=U_{\text {pre }, g} \mid I_{\text {pre }, 3} ;$ con jornada laboral precaria $D=U_{\text {pre }, g} \mid I_{\text {pre }, 4}$; y los trabajadores sin seguridad social $E=U_{\text {pre, } g} \mid I_{p r e, 5}$. En todos los casos, $I_{p r e, i}$ es una función indicador que toma el valor de uno si los trabajadores con precariedad extrema general cumplen con la condición de tener una de las $i=1,2,3,4,5$ condiciones de la precariedad extrema particular definidas (véase el Cuadro 1). 
Con base en las cinco condiciones de precariedad laboral extrema, se definieron los niveles y subniveles de precariedad laboral extrema. El resultado de la organización de los niveles indica la existencia de un nivel cero (0), que es aquel en donde los trabajadores mantienen la condición de precariedad sólo por tener ingresos mensuales precarios extremos; pero están sindicalizados, tienen un contrato no precario, cuentan con prestaciones, laboran una jornada completa (entre 35 y 48 horas) y cuentan con seguridad social; por ello están en el nivel más bajo de precariedad laboral extrema. En nivel 1 de precariedad laboral se identifican los trabajadores con precariedad extrema general o con ingreso precario extremo y que tienen una condición de precariedad laboral extrema en las cinco posibilidades. En el nivel de precariedad 2 se identifican los trabajadores que cumplan con tener dos condiciones de precariedad laboral extrema, y así sucesivamente, hasta el nivel 5. Para la identificación de los niveles de precariedad laboral extrema es importante señalar que lo relevante es el número de combinaciones de las condiciones laborales, sin importar cuál de ellas sea. En este caso, el orden de la valoración de los niveles de precariedad laboral extrema es muy claro: entre mayor es el nivel, los trabajadores tienden a tener peores condiciones de precariedad laboral extrema. Esto es, $\mathrm{Nivel}_{0}<\mathrm{Nivel}_{1}<\mathrm{Nivel}_{2}<\mathrm{Nivel}_{3}$ $<\mathrm{Nivel}_{4}<\mathrm{Nivel}_{5}$, que en términos de la variable numérica que ordena las condiciones queda como $0,1,2,3,4$ y 5 .

En el caso de los subniveles de precariedad laboral extrema, son relevantes tanto el número de condiciones como el tipo de combinación entre ellas. El primero de los subniveles tiene un solo elemento del nivel 0 (Subnivel $\left._{0} 1_{1}>\ldots\right)$, que son los trabajadores con precariedad laboral por ingreso precario extremo. En el siguiente nivel, y considerando la variedad de aspectos económicos y sociales, establecimos la valoración de las cinco condiciones individuales de precariedad extrema, lo cual implica que, en el caso de los subniveles del nivel 1 (véase el Cuadro 1), se ordenaran de la menos mala a la peor condición de precariedad, con la siguiente regla: los trabajadores sin sindicato son menos precarios que los trabajadores con jornada precaria, que a su vez son menos precarios que los trabajadores sin prestaciones; éstos son menos precarios que los trabajadores sin seguridad social, $\mathrm{y}$ todos son menos precarios que los trabajadores con contrato precario. En términos de los cinco subniveles del nivel 1 de precariedad quedan como: Subnivel $_{1 \_1}>$ Subnivel $_{1 \_4}>$ Subnivel $_{1 \_3}<$ Subnivel $_{1 \_5}>$ Subnivel $_{1 \_2}$. Estos cinco subniveles tienen como correspondientes los valores numéricos de orden de 1,2,3,4 y 5. La ventaja de esta regla de los primeros subniveles y su valoración es que permite ordenar todos los subniveles de precariedad subsiguientes con la simple multiplicación de los valores de las condiciones. 


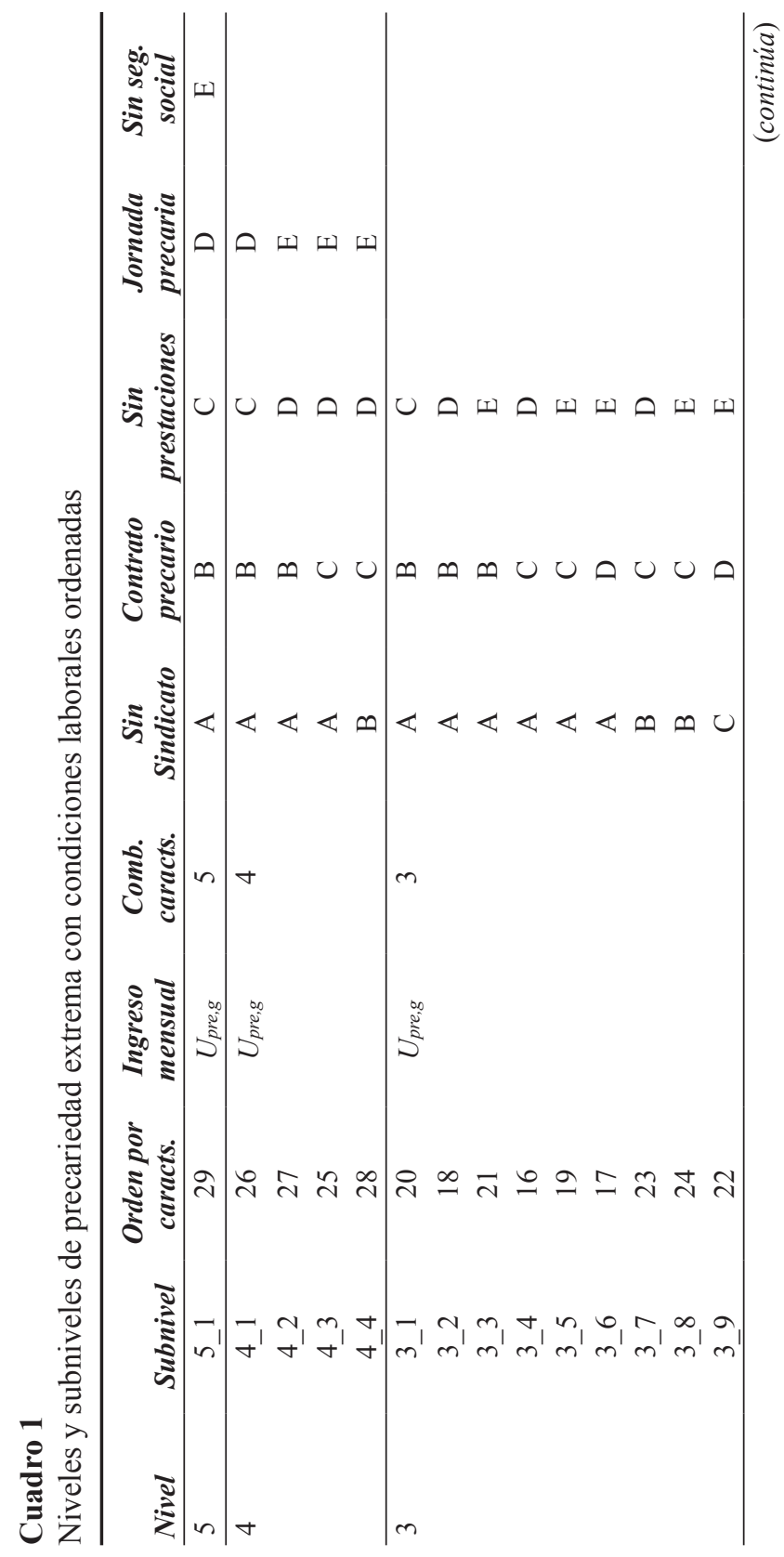




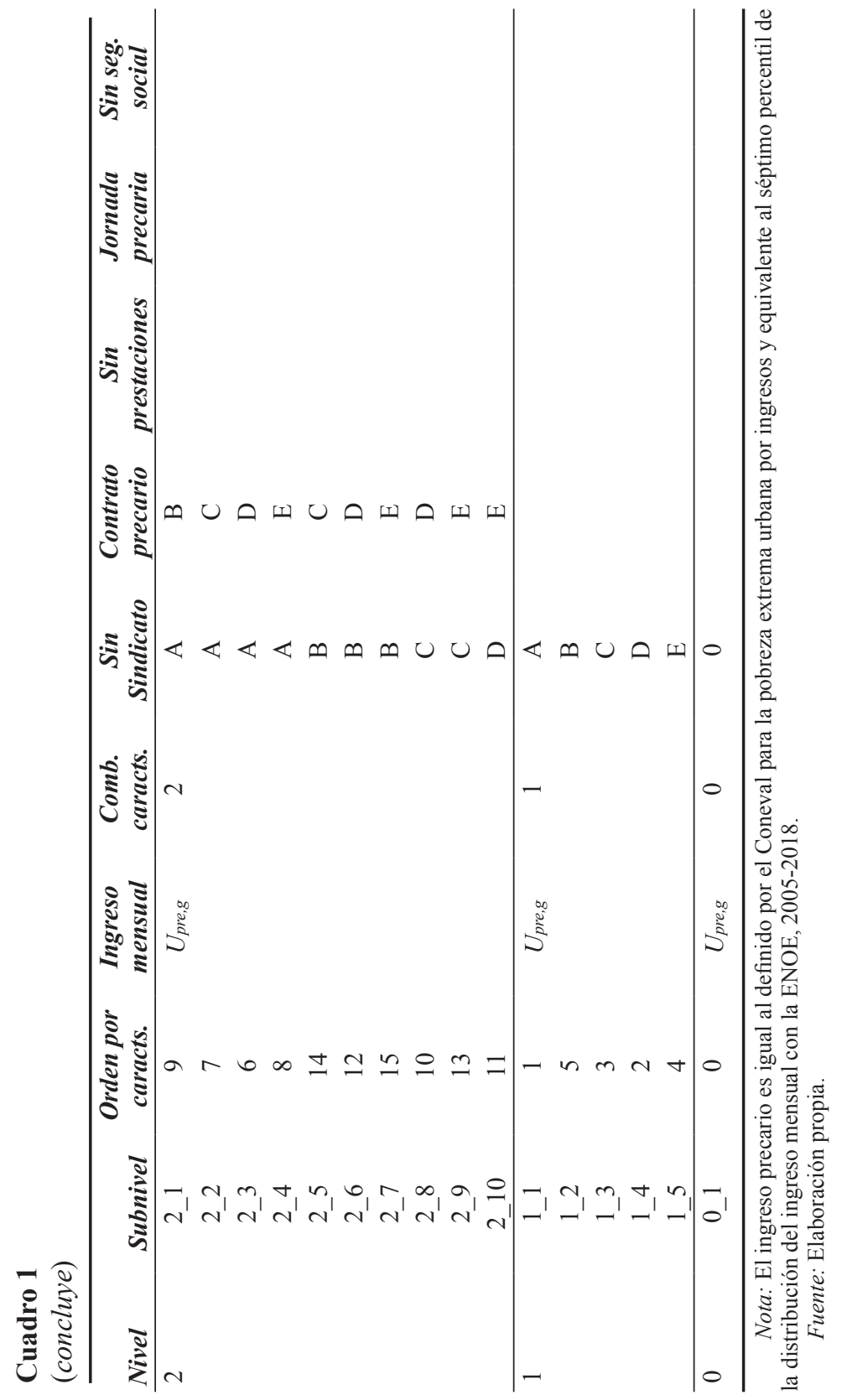


Por lo anterior, para los trabajadores con dos condiciones de precariedad extrema, que define los diez subniveles del nivel 2, se establece que aquellos sin sindicato y jornada laboral precaria se encuentran en Subni$v l_{2}{ }_{3}$ más bajo de precariedad, mientras que la peor condición de precariedad extrema la tienen los trabajadores con contrato precario y sin seguridad social que definen el Subnivel 27 . El orden de todos los subniveles del orden dos es: Subnivel $2_{3}>$ Subnivel $_{2}{ }_{2}>$ Subnivel $_{2}{ }_{4}<$ Subnivel $_{2}{ }_{1}>$ Subnivel $_{2} 8$

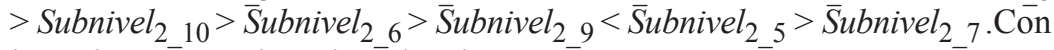
los valores numéricos de orden de $6, \ldots, \overline{1} 15$.

Los trabajadores con tres características se ordenaron en nueve combinaciones, donde la condición más baja de precariedad laboral extrema son aquellos sin sindicato, sin prestaciones, y jornada precaria (Subnivel ${ }_{3} 4$ ); y los trabajadores con la peor condición de precariedad son los que tienen contrato precario, no tienen prestaciones ni seguridad social ( Subnivel $_{3}$ 8). En este caso la lista con el orden de los nueve subniveles de orden es: Su $\bar{b}$ nivel $_{3}{ }_{4}>$ Subnivel $_{3 \_} 6$ Subnivel $_{3 \_}<$Subnivel $_{3}{ }_{5}>$ Subnivel $_{3 \_}>$Subni- $_{\text {S }}$ vel $_{3}{ }_{3}^{-}>$Subnivel $_{3}{ }_{9}^{-}$Subnivel $_{3}{ }_{7}^{-}<$Subnivel $_{3}{ }_{8}$. Y los valores numéricos van de 16 hasta 24 .

La condición más baja para los cuatro subniveles del nivel 4 fueron los trabajadores que no tienen sindicato, sin prestaciones, con jornada precaria y sin seguridad social, hasta el último grupo, donde los trabajadores tienen contrato precario, sin prestaciones, jornada precaria y sin seguridad social. El orden de los subniveles es: Subnivel $_{4 \_}>$Subnivel $_{4}{ }_{1}>$ Subnivel $_{4 \_}<$ Subnivel $_{4}$, con numeración correspondiente de 25 hastā 28.

Por último, se tiene el subnivel donde los trabajadores con cinco condiciones a la vez se consideran con las peores condiciones de precariedad laboral extrema. En tal caso, los individuos aceptan trabajar sin sindicato, con contrato precario, sin prestaciones, jornada precaria y sin seguridad social (Subnivel S_1 $_{\text {); }}$ a este subnivel se le asignó el valor número 29. Al final se tiene a diferentes grupos de trabajadores clasificados en 30 subniveles de precariedad y ordenados de menor a mayor precariedad con la asignación numérica de 0 a 29 (véase el Cuadro 1).

\subsection{Tendencias de los niveles y subniveles de precariedad laboral extrema, 2005-2018}

Con la aplicación de la metodología de grupos, en primer lugar, se identificó el perfil sociodemográfico de los trabajadores con precariedad laboral extrema, con su nivel de escolaridad, experiencia, capacitación, edad, sexo, 
si es casado, jefe del hogar y si tiene apoyos de programas sociales. La escolaridad promedio de los empleados precarios extremos fue 6.41 años, que es equivalente a la primaria completa; los años de experiencia de los trabajadores fueron de casi una década (9.84); en promedio dedicaron 2 horas con 58 minutos a los estudios o la capacitación. La edad promedio de los trabajadores fue de 39.3 años; el $45 \%$ de los trabajadores son hombres o predomina $55 \%$ de mujeres; el $65 \%$ son casados, pero solamente el $42 \%$ son jefes de hogar; y, finalmente, sólo el $25 \%$ de los trabajadores manifestaron recibir apoyos de programas sociales (véase el Cuadro 4 sobre los efectos marginales).

En segundo lugar, con la metodología de grupos se estimó que el empleo precario alcanzó los 2.6 millones de trabajadores en 2018 y, aunque es ligeramente mayor al observado en 2005 (2.0), muestra dos aspectos importantes antes y después de la crisis de 2009. En el primer periodo, de 2005 a 2008 , el empleo precario extremo se redujo en $40 \%$, de 2.0 a 1.2 millones de personas, y se relaciona directamente con la dinámica económica del periodo y sobre todo con el comportamiento económico de 2007; en ese año la economía creció en 5\%. Con la crisis económica de 2009 se inicia una nueva fase con una tendencia creciente en la creación de empleos con condiciones de precariedad extrema; durante la crisis se crearon $86 \%$ más empleos con precariedad extrema y $2.8 \%$ en promedio de 2010 a 2018 (véase el Cuadro 2).

En estudios como los de García (2010) se señala que existe un canal de vinculación entre el desempleo y el empleo precario, por la incapacidad de la economía mexicana de generar empleos al mismo ritmo en que crece la población en edad de trabajar (Ruiz y Ordaz, 2011) y en condiciones de empleo decentes (Weller, 2017). El incremento de los niveles de desempleo, junto a la extensión de la inseguridad laboral y la desprotección social, no sólo revela un debilitamiento continuo de la relación entre crecimiento económico y empleo, sino que cuestiona las potencialidades del modelo económico actual para absorber fuerza de trabajo, y reducir la pobreza y las desigualdades persistentes y crecientes (Bayón, 2006).

La estimación del empleo precario extremo nos muestra que su magnitud es parecida al nivel del desempleo; para compararlos se calcularon las proporciones con respecto a la población ocupada de 2005-2018. Como se observa en la Gráfica 3, existen dos tendencias importantes: antes y después de la crisis de 2009. En el primer periodo, la tasa de precariedad extrema se caracterizó por ser mayor al desempleo, con una tendencia negativa y de igualación a la tasa de desempleo. Con la crisis de 2009, las tasas de precariedad extrema y desempleo aumentaron y prácticamente se mantuvieron en el mismo nivel (5.7\%) hasta 2012. Posterior a ello, la tasa de desempleo 


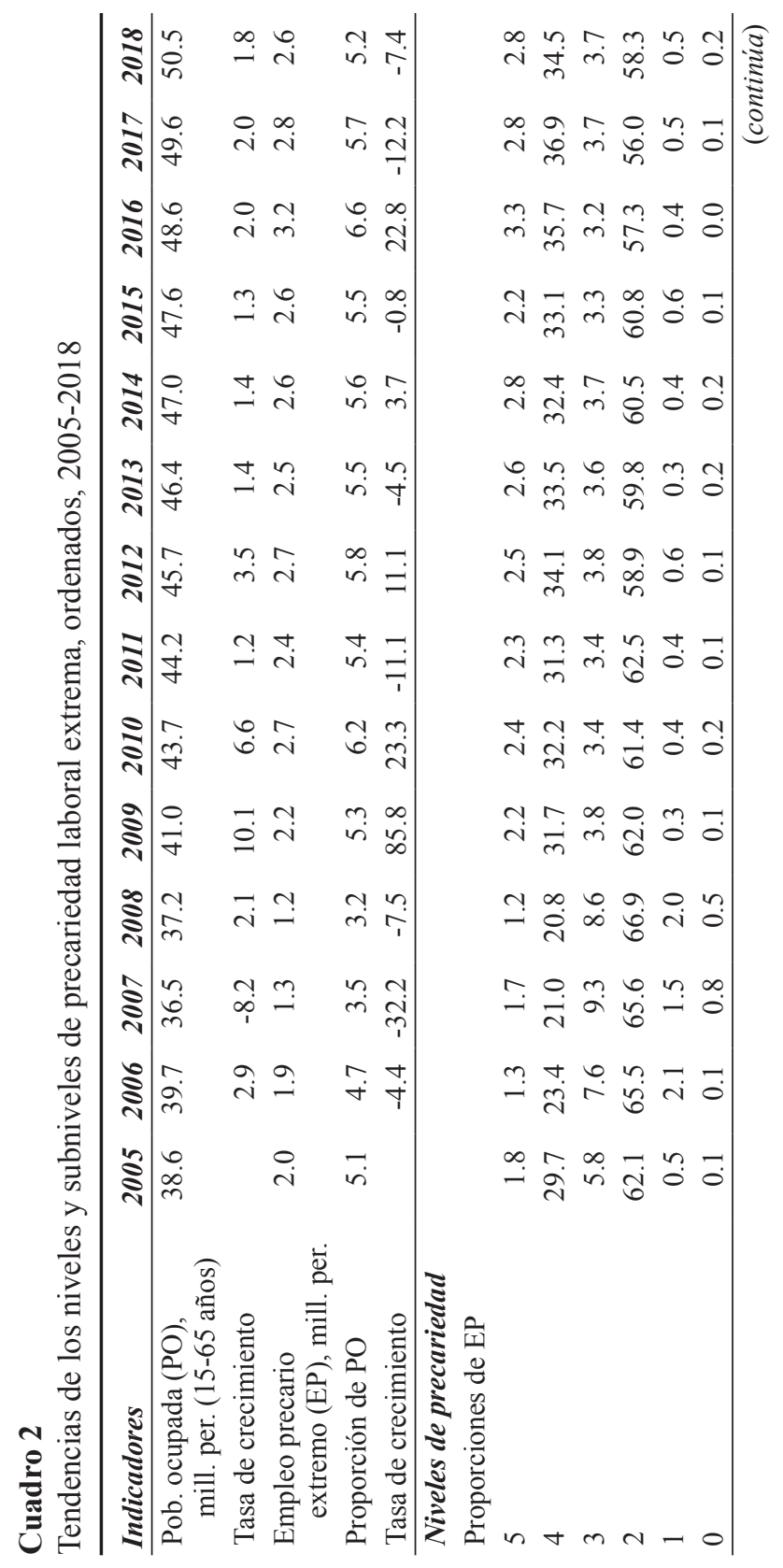




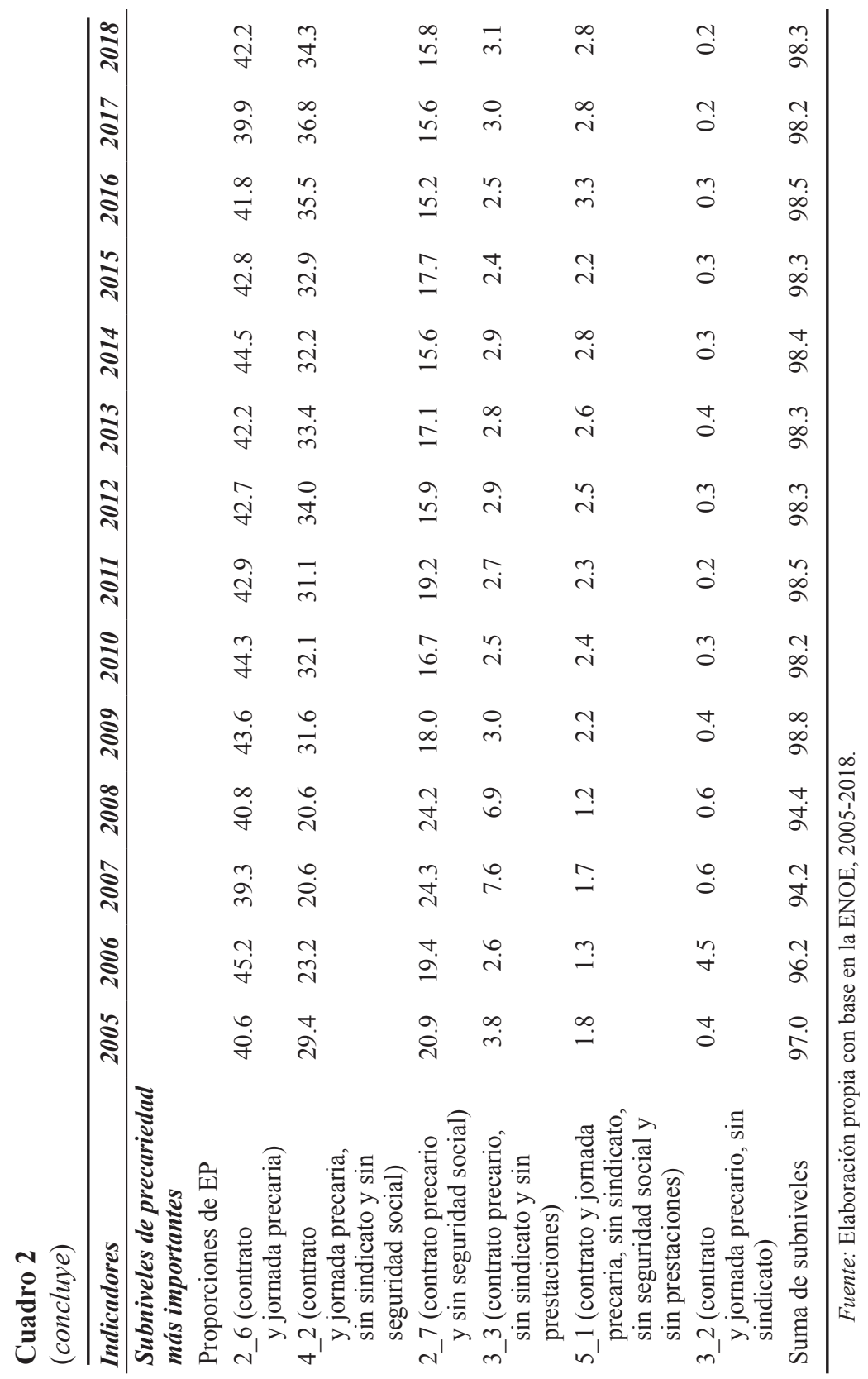


se ha reducido con el mejor desempeño económico, mientras que la tasa de empleo precario extremo se mantuvo prácticamente en el mismo nivel, con la excepción del aumento de 2016. Este hecho es muy importante debido a que muestra cómo el empleo precario extremo depende menos del ciclo económico y se explica por factores vinculados a la política pública instrumentada desde 2009, por lo que la generación de empleo en general y precario extremo en particular, depende de la oferta laboral y su composición, así como de los factores que determinan la demanda laboral (Weller, 2000).

\section{Gráfica 3}

Tasas de precariedad laboral extrema y desempleo. Proporciones con respecto a la población ocupada

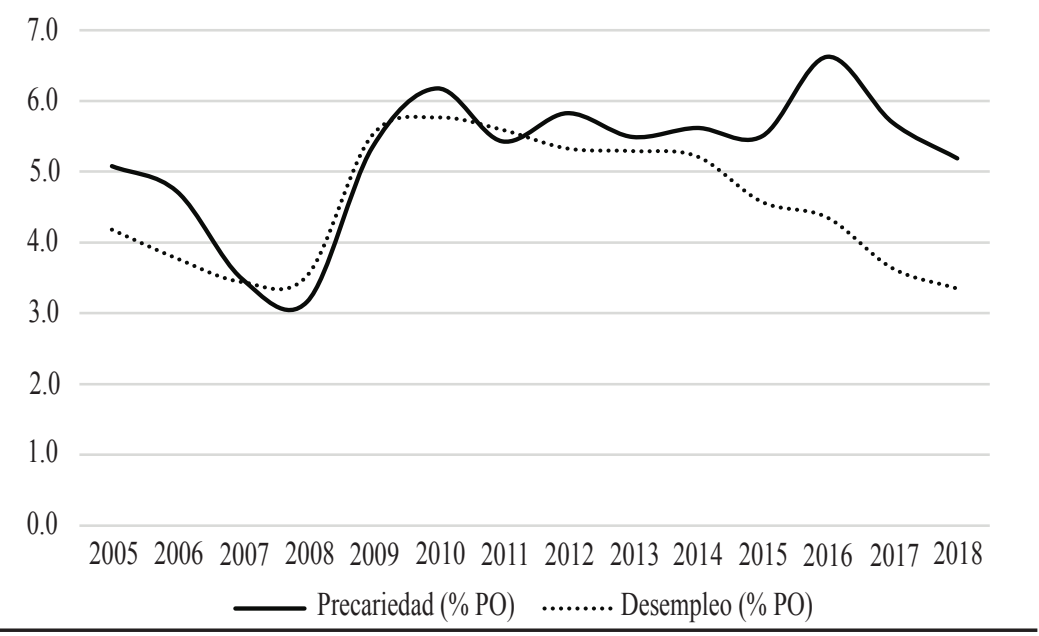

Fuente: Elaboración propia con base en la ENOE, 2005-2018.

Del grupo de empleo precario general, se identificaron los grupos de trabajadores con los niveles de precariedad de 0 hasta 5 , de acuerdo con la combinación del número de condiciones de precariedad extrema (Gráfica 4). Nuestras estimaciones muestran que el nivel de precariedad extrema que predomina en la economía mexicana es el de trabajadores con ingresos de precariedad extrema y con dos condiciones de precariedad extrema de las cinco identificadas (contrato precario, no tienen prestaciones, no tienen una jornada completa y no cuentan con seguridad social). En el nivel 2 de precariedad laboral extrema se concentraron 1.2 millones de trabajadores en 
2005 y, aunque con una reducción previa a la crisis de 2009, se han mantenido alrededor de 1.6 millones en el periodo 2010-2018. En este nivel 2 de precariedad extrema se concentraron entre 56 y $68 \%$ de los empleados precarios en el periodo (véase el Cuadro 2 y la Gráfica 4). El siguiente nivel de precariedad laboral extrema que predomina en la economía mexicana es donde se combinan cuatro condiciones de precariedad extrema, y se estimó en 903 mil empleos en 2018, con un mínimo de 245 mil empleos precarios extremos en 2008, y con clara tendencia creciente a partir de la crisis de 2009. En el nivel 4 de precariedad extrema se concentraron entre el 21 y el $37 \%$ de los empleados precarios extremos, y el aumento se debe principalmente a la reducción del nivel 3 (véase el Cuadro 2 y las Gráficas 4 y 5). En los niveles de precariedad laboral 1, 3 y 5 se calcularon los menores números de trabajadores, por lo que son los que menos predominan en la economía mexicana. Es importante mencionar que el nivel 1 de precariedad laboral es el menos predominante.

\section{Gráfica 4}

Tendencias de los niveles de precariedad laboral extrema ordenados.

Millones de personas

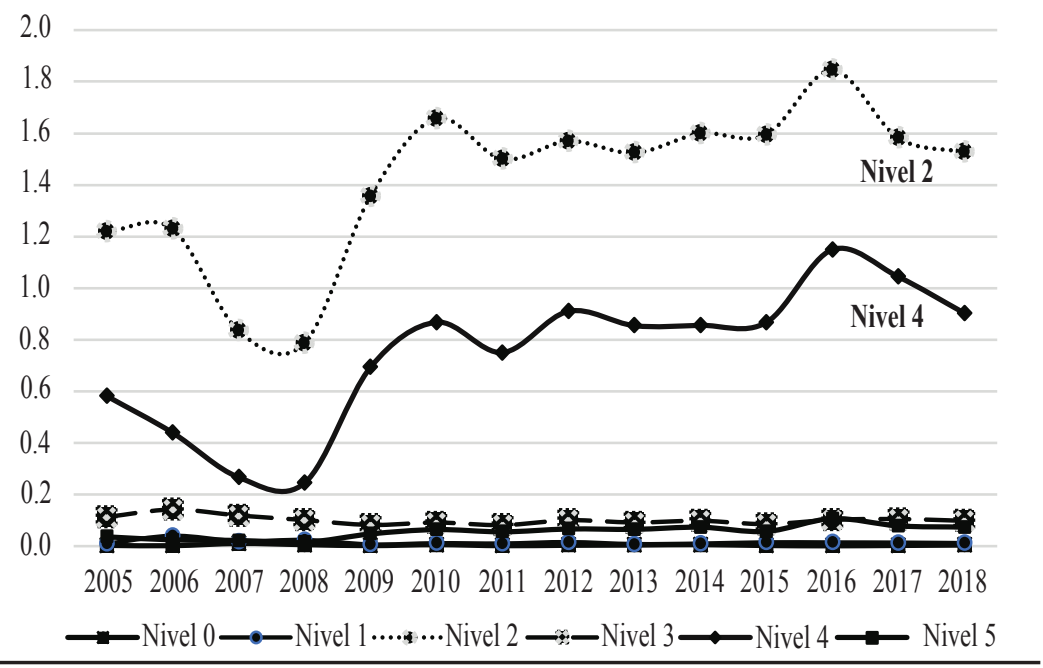

Fuente: Elaboración propia con base en la ENOE. 


\section{Gráfica 5}

Distribución de los niveles de precariedad laboral extrema ordenados. Proporción del empleo con precariedad laboral extrema total

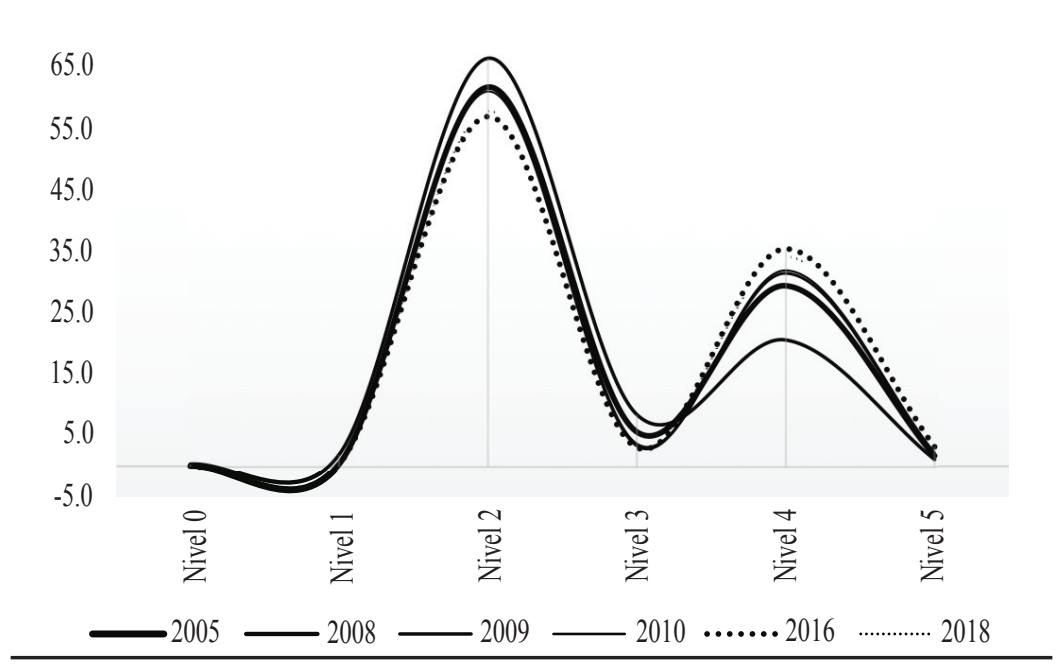

Fuente: Elaboración propia con base en la ENOE.

Los resultados anteriores muestran niveles de precariedad laboral extrema concentrados principalmente en los casos con dos y cuatro condiciones, con tendencias persistentes en los trece años de análisis. Con los subniveles de precariedad laboral extrema se identificaron, con mayor detalle, cuáles son las combinaciones de condiciones que predominan en los niveles 2 y 4, principalmente. En el Cuadro 2 se presentan las proporciones de los seis principales subniveles de precariedad laboral extrema que suman más del 98\%; y en la Gráfica 6 se muestran las distribuciones de todos los subniveles de precariedad extrema. En el Subnivel ${ }_{2-6}$, con valor numérico de orden 12 (Gráfica 6), se concentra la proporción más alta, entre 40 a 45\%, del empleo precario extremo que indica que la combinación de contrato y jornada precaria es la condición que más predomina en el mercado laboral mexicano. El segundo subnivel predominante son los trabajadores con las siguientes cuatro condiciones de precariedad laboral extrema: contrato y jornada precaria, sin sindicato y sin seguridad social; este Subnivel $_{42}$ tiene un valor numérico alto de precariedad extrema (27) (véase el Cuadro 2 y la Gráfica 6). El Subnivel $_{27}$ de precariedad laboral extrema es el tercero más importante, con combinación de las condiciones de contrato precario y sin seguridad social. 


\section{Gráfica 6}

Distribución de los subniveles de precariedad laboral extrema ordenados. Proporción del empleo con precariedad laboral extrema total

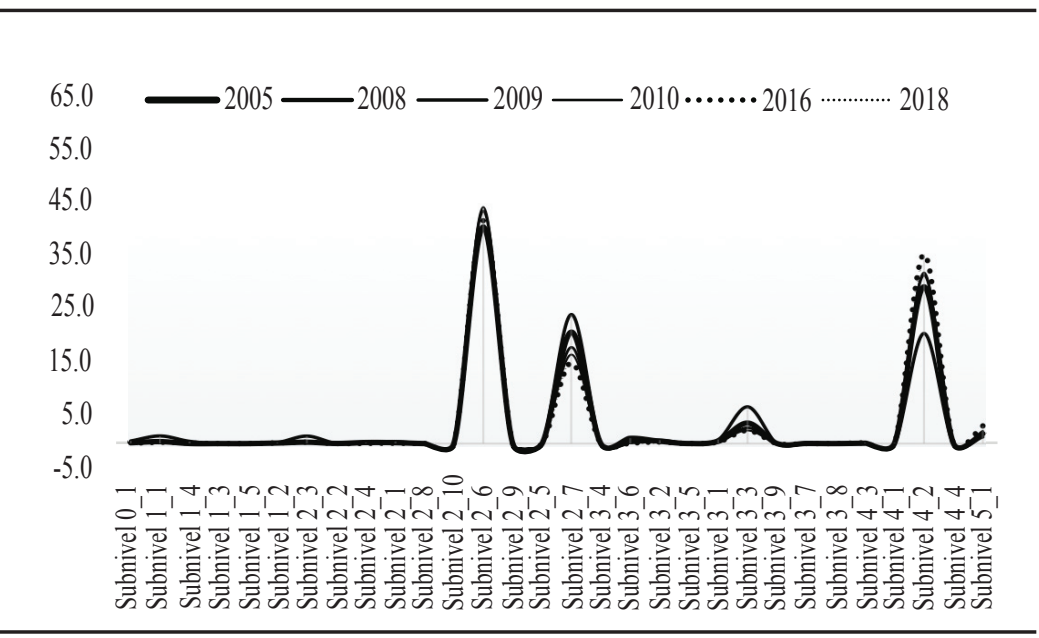

Fuente: Elaboración propia con base en la ENOE.

Entre los subniveles de precariedad laboral más relevantes que se presentan en el Cuadro 2, pero que en proporción son menores al 10\% del empleo precario extremo, se encuentra el Subnivel ${ }_{33}$, que es la combinación de las condiciones de contrato precario, sin sindicato y sin prestaciones; éste resulta interesante por su tendencia creciente en el periodo 2005-2008, al pasar de una proporción de 3.8 a $7.0 \%$ del empleo precario extremo, pero después de la crisis de 2009 cambió a una tendencia decreciente hacia 2018. Este comportamiento se observa como una tendencia de reducción de la curva de participación en el subnivel con valor numérico de 21 en la Gráfica 6. Un caso especial es el identificado por el Subnivel $_{51}$, donde se combinan todas las condiciones de precariedad extrema al mismo tiempo; ha fluctuado entre 35 mil y 70 mil trabajadores, que representan el 1.2 y $3.3 \%$ del empleo precario extremo en los años 2006 y 2018. Finalmente, el último grupo de trabajadores con precariedad extrema interesante que se presenta en el Cuadro 2, son los del Subnivel ${ }_{32}$, que combina el contrato y la jornada precarios y sin sindicato; son menos del 1\% del empleo precario extremo y tuvieron un comportamiento atípico en 2006, el cual se observa claramente en el subnivel con valor 18 en la Gráfica 6. 
Con estos resultados por subnivel de precariedad laboral extrema queda claro que la tendencia principal en el periodo 2005-2018 ha sido que buena parte de los empleos precarios extremos tienen la combinación de condiciones concentradas en ingresos precarios extremos, contrato precario, jornada precaria y sin protección social; son trabajadores sin acceso a servicios médicos suficientes y de calidad. Por otro lado, las condiciones de precariedad laboral extrema sin sindicato y sin prestaciones son las que menos ocurren o se presentan en la determinación de las tendencias del empleo con precariedad laboral extrema en el mercado laboral mexicano.

\section{Análisis de los resultados del modelo probit-pool con condiciones ordenadas de precariedad laboral extrema}

En esta parte se presentan los resultados de la estimación y el análisis de dos modelos probit-pool con condiciones de precariedad laboral extrema ordenadas como en la ecuación $Y_{i, t}^{*}=F\left(X_{i, t} \beta\right)+u_{i, t}$, donde la variable latente $Y_{i, t}^{*}$ se define con los niveles de precariedad laboral extrema $(0,1, \ldots, 5)$ para el primer modelo, y con los subniveles de precariedad laboral extrema $(0,1, \ldots, 29)$ para el segundo modelo probit-pool, por cada individuo $i$ y $t=2005, \ldots, 2018$, año de la ENOE. En los dos modelos se utiliza un conjunto de condicionantes o variables explicativas $\left(X_{i, t}\right)$ que ayuda a entender la probabilidad de ocurrencia de cada nivel y subnivel de precariedad laboral extrema. El primer conjunto de variables se define para probar si con mayor escolaridad, experiencia y capacitación existe una menor probabilidad de ocurrencia por niveles y subniveles de precariedad laboral extrema. En el segundo conjunto de variables se utiliza el perfil sociodemográfico del trabajador: edad, sexo, si es casado, es jefe del hogar y si recibe apoyos de programas sociales. Por último, se utiliza el contexto urbano y rural para analizar la influencia de la estructura territorial, así como el tamaño de la empresa y el sector económico en que labora para medir las restricciones sobre la demanda laboral.

\subsection{Datos y variables explicativas}

Las variables explicativas que se utilizaron en los modelos probit-pool ordenados se construyeron con los microdatos de la ENOE de 2005 a 2018. Las tres variables que identifican el nivel educativo de los trabajadores son continuas: los años de escolaridad se construyeron considerando estudios 
terminados de primaria, secundaria, secundaria técnica, preparatoria, preparatoria con carrera técnica, licenciatura, maestría y doctorado. Para la capacitación se consideraron las horas dedicadas al estudio y la capacitación; y, finalmente, la experiencia se construyó con el año en que se comenzó a trabajar por primera vez. La edad de los trabajadores es también un variable continua, mientras que las variables dicotómicas fueron: sexo (1 hombre y 0 mujeres), 1 si es casado, 1 si es jefe de hogar, 1 si se localiza en la parte urbana y 1 si recibe apoyos de programas sociales. Para los casos de tamaño de empresa, sector económico y año de ENOE, se utilizaron variables categóricas o dicotómicas múltiples, donde la primera categoría se utiliza como base de comparación: para el tamaño de empresa, 1 es para la micro (base de comparación), 2 para la empresa pequeña, 3 la mediana y 4 la empresa grande. En cuanto a los tres sectores económicos, 1 es para el primario, 2 en el secundario y 3 en servicios; finalmente para la variable año de la ENOE, 2005 se considera el año base, 2 para 2006,... hasta 15 para 2018.

\subsection{Resultados econométricos de los modelos probit-pool con niveles y subniveles de precariedad laboral extrema ordenados}

En los modelos probit-pool que se estimaron se utilizó como variable latente $Y_{i, t}^{*}$ los niveles de precariedad laboral extrema, con valores numéricos ordenados de $0,1, \ldots, 5$, los subniveles de precariedad laboral extrema de $0,1, \ldots, 29$; y las variables explicativas $\left(X_{i, t}\right)$ : escolaridad, experiencia, capacitación, edad, sexo, casado, jefe del hogar, urbano o rural, apoyo de programas sociales, tamaño de empresa, sector económico y los años de la ENOE. Los dos modelos probit-pool para los niveles y subniveles de precariedad laboral extrema ordenados se estimaron para el pool de indicadores por individuos y el periodo 2005-2018. En el Cuadro 3 se presentan los resultados econométricos de los dos modelos y, como se esperaba para este tipo de modelos, la pseudo- $R^{2}$ presenta valores muy pequeños, entre $0.09 \mathrm{y}$ 0.07 para niveles y subniveles respectivamente. Lo relevante de los resultados econométricos es que todas las variables explicativas son altamente significativas.

En cuanto a los resultados analíticos, el signo de los coeficientes de las variables educación y experiencia indican que, a mayor educación y experiencia, menor probabilidad de presentar niveles y subniveles altos de precariedad laboral extrema. Aunque el coeficiente de la variable capacitación también es estable, el signo positivo indica que los trabajadores que dedican algunas horas a la capacitación y/o seguir estudiando, tienen una mayor pro- 
babilidad de estar en una situación de mayor nivel o subnivel de precariedad laboral extrema (Cuadro 3).

Del grupo de indicadores que muestran el perfil socioeconómico, el signo negativo de los coeficientes implica que los trabajadores en condiciones de precariedad extrema que son hombres y jefes del hogar tienen menor probabilidad de localizarse en niveles o subniveles de precariedad laboral extrema más altos. Mientras que el signo positivo de los coeficientes indica que los trabajadores con mayor edad, casados y con apoyos de programas sociales tendrían mayor probabilidad de encontrarse en una situación de alto nivel o subnivel de precariedad laboral extrema.

\section{Cuadro 3}

Modelos probit-pool con niveles y subniveles de precariedad laboral extrema ordenados, explicados por X, 2005-2018

\begin{tabular}{|c|c|c|c|c|}
\hline \multirow[b]{3}{*}{ Variables $(X)$} & \multicolumn{4}{|c|}{ Precariedad extrema } \\
\hline & \multicolumn{2}{|c|}{ Niveles } & \multicolumn{2}{|c|}{ Subniveles } \\
\hline & Coef. & Prob $>z$ & Coef. & Prob $>z$ \\
\hline Escolaridad & -0.017 & 0.00 & -0.018 & 0.00 \\
\hline Experiencia & -0.010 & 0.00 & -0.010 & 0.00 \\
\hline Capacitación & 0.011 & 0.00 & 0.010 & 0.00 \\
\hline Edad & 0.004 & 0.00 & 0.003 & 0.00 \\
\hline Sexo & -0.127 & 0.00 & -0.129 & 0.00 \\
\hline Casado & 0.056 & 0.00 & 0.053 & 0.00 \\
\hline Jefe del hogar & -0.081 & 0.00 & -0.093 & 0.00 \\
\hline Urbano / rural & 0.039 & 0.00 & 0.035 & 0.00 \\
\hline Apoyo prog. sociales & 0.118 & 0.00 & 0.116 & 0.00 \\
\hline \multicolumn{5}{|l|}{ Tamaño empresa } \\
\hline \multicolumn{5}{|l|}{ Micro (base) } \\
\hline Pequeña & -0.495 & 0.00 & -0.540 & 0.00 \\
\hline Mediana & -0.945 & 0.00 & -1.018 & 0.00 \\
\hline Grande & -1.236 & 0.00 & -1.295 & 0.00 \\
\hline \multicolumn{5}{|l|}{ Sector económico } \\
\hline \multicolumn{5}{|l|}{ Primario (base) } \\
\hline Secundario & -0.119 & 0.00 & -0.134 & 0.00 \\
\hline Terciario & 0.039 & 0.00 & 0.015 & 0.00 \\
\hline
\end{tabular}




\begin{tabular}{|c|c|c|c|c|}
\hline \multirow[b]{2}{*}{ Año de ENOE } & \multicolumn{2}{|c|}{ Niveles } & \multicolumn{2}{|c|}{ Subniveles } \\
\hline & Coef. & Prob $>z$ & Coef. & Prob $>z$ \\
\hline \multicolumn{5}{|l|}{2005 (base) } \\
\hline 2006 & 0.044 & 0.00 & 0.056 & 0.00 \\
\hline 2007 & -0.712 & 0.00 & -0.671 & 0.00 \\
\hline 2008 & -0.720 & 0.00 & -0.688 & 0.00 \\
\hline 2009 & 0.126 & 0.00 & 0.136 & 0.00 \\
\hline 2010 & 0.161 & 0.00 & 0.170 & 0.00 \\
\hline 2011 & 0.176 & 0.00 & 0.188 & 0.00 \\
\hline 2012 & 0.170 & 0.00 & 0.183 & 0.00 \\
\hline 2013 & 0.179 & 0.00 & 0.188 & 0.00 \\
\hline 2014 & 0.191 & 0.00 & 0.201 & 0.00 \\
\hline 2015 & 0.158 & 0.00 & 0.163 & 0.00 \\
\hline 2016 & 0.282 & 0.00 & 0.288 & 0.00 \\
\hline 2017 & 0.167 & 0.00 & 0.179 & 0.00 \\
\hline 2018 & 0.191 & 0.00 & 0.206 & 0.00 \\
\hline Pseudo $R^{2}$ & \multicolumn{2}{|c|}{0.09} & \multicolumn{2}{|c|}{0.07} \\
\hline
\end{tabular}

Fuente: Elaboración propia con base en programación Stata 13.

En cuanto a las variables de contexto, el coeficiente positivo de la variable urbano-rural muestra que existe una mayor probabilidad de localizar empleo con mayores niveles y subniveles de precariedad laboral extrema en zonas urbanas (véase el Cuadro 3). Para el tamaño de empresa se estimaron signos negativos para todos los tamaños y numéricamente más altos en empresas grandes. Estos resultados muestran que, en comparación con las empresas micro, existe una mayor probabilidad de localizar trabajadores con menores niveles y subniveles de precariedad laboral extrema en empresas pequeñas, medianas y grandes. El signo negativo para el sector secundario y positivo para el terciario, indica que los trabajadores del secundario tienen una menor probabilidad y el sector servicios una mayor probabilidad de pertenecer a grupos de empleos con mayores niveles y subniveles de precariedad laboral extrema, en comparación con los trabajadores del sector primario. Por último, la variable de año de ENOE muestra, en referencia a 2005 , la reducción de hasta $72 \%$ del promedio del empleo en niveles y subniveles de precariedad laboral extrema en 2008, el cambio de comportamiento en 2009 y la tendencia hacia un crecimiento de $19 \%$ en 2018. 


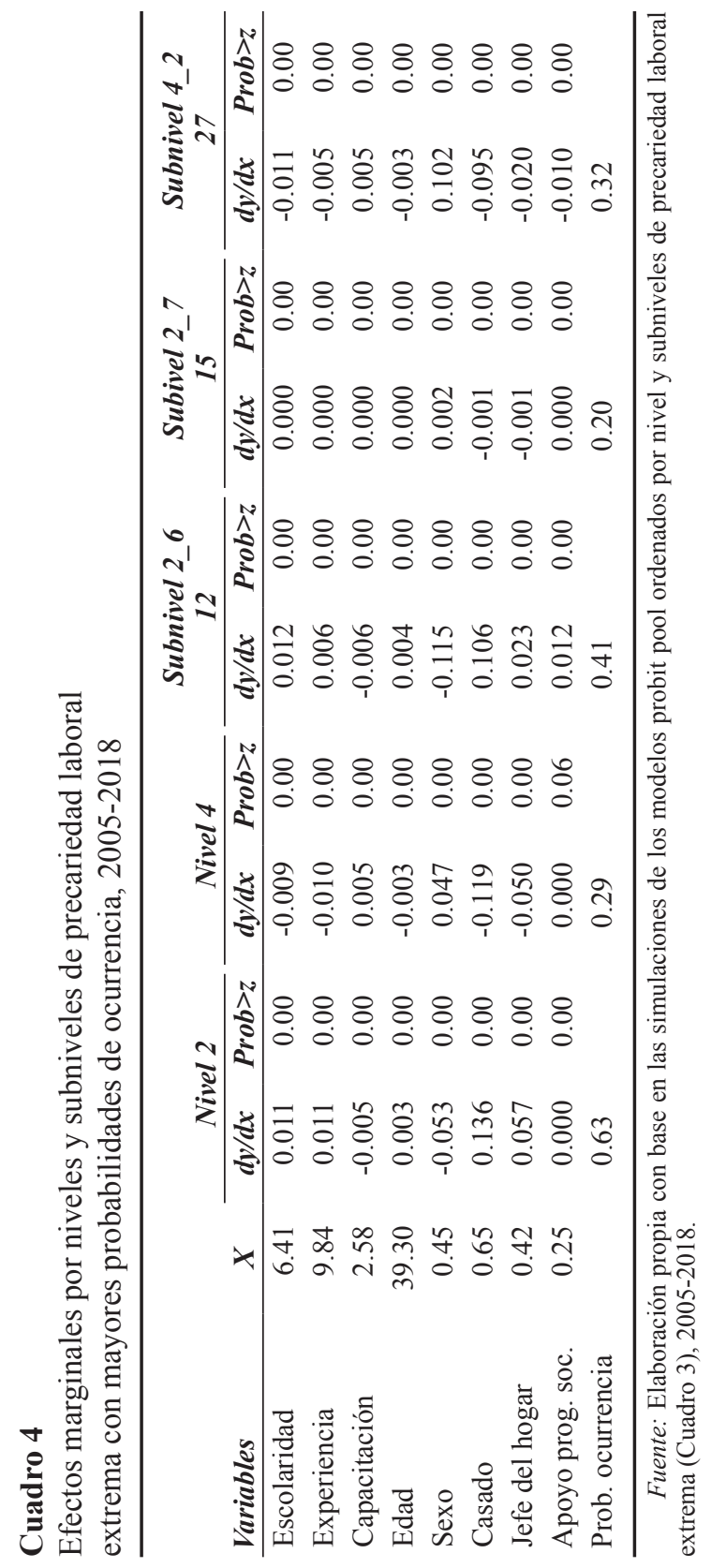




\subsection{Análisis de los efectos marginales por niveles y subniveles de precariedad laboral extrema}

Con los efectos marginales se cuantifica cómo afecta un aumento unitario de cada variable independiente en el aumento o disminución de la probabilidad de menores a mayores niveles o subniveles de precariedad laboral extrema. El tamaño de los efectos marginales depende de la magnitud y signo de los coeficientes, y de los valores iniciales de las variables exógenas de cada individuo en la función de densidad. Para el análisis de los efectos marginales se elaboraron las simulaciones para cada uno de los niveles $(0, \ldots, 5)$ y subniveles $(0, \ldots, 29)$ de precariedad laboral, de acuerdo con los modelos probit-pool ordenados estimados para 2005, 2008, 2009 y 2015. En cada simulación se obtuvo la probabilidad de ocurrencia para cada nivel y subnivel de precariedad laboral, las cuales fueron consistentes con las distribuciones que se presentaron en las Gráficas 5 y 6 . Esto es, los niveles de precariedad laboral extrema en 2 y 4 y los subniveles en 12 (2_6), 27 (4_2) y 15 (2_7) concentraron las mayores probabilidades de ocurrencia.

En el caso de los efectos marginales de los dos niveles de precariedad laboral (véase el Cuadro 4), sumaron el 92\% de las probabilidades de ocurrencia en promedio para el periodo 2005-2018 de las simulaciones; el 63\% de tales probabilidades se concentraron en el Nivel 2 y $29 \%$ en el Nivel 4. Es importante destacar que las probabilidades de ocurrencia del empleo con precariedad laboral extrema en el Nivel 2 se mantuvieron, mientras las del Nivel 4 aumentaron ligeramente sobre todo posteriormente a la crisis económica de 2009 (véase el Cuadro 2). Con las simulaciones para los subniveles, se encontró que el $93 \%$ de las probabilidades de ocurrencia se concentraron en promedio en tres subniveles de precariedad laboral; dos de ellas del Nivel 2 y uno del Nivel 4 (véase el Cuadro 4). Por orden de importancia, el Subnivel 2_6 tiene el $41 \%$ de las probabilidades de ocurrencias, en el segundo lugar el Subnivel 4_2 con 32\% de las probabilidades de ocurrencias, y finalmente el Subnivel 2_7 con el 20\% de las probabilidades de ocurrencia, en promedio para todo el periodo de análisis 2005-2018.

Con los resultados de las probabilidades de ocurrencia, se confirma que la precariedad laboral extrema en México se concentra prácticamente en dos niveles: en el primero, el 63\% de los trabajadores tienen ingresos precarios extremos y además presentan dos de las cinco condiciones de precariedad laboral extrema (sin sindicato, con contrato precario, sin prestaciones, con jornada laboral precaria y sin seguridad social). Mientras que en el segundo grupo de mayor precariedad laboral extrema se concentra el 
$29 \%$ de los trabajadores con ingresos precarios, y además presentan cuatro de las cinco características de precariedad laboral extrema. En primer lugar, los trabajadores con ingresos precarios extremos, con contrato y jornada laboral precaria (Subnivel ${ }_{26}$ y orden 12; véase el Cuadro 4) representan la mayor probabilidad de ocurrencia del empleo con precariedad laboral extrema; en segundo lugar, son los trabajadores con ingresos precarios extremos, con contrato y jornada precaria, sin seguridad social y sin sindicato (Subni$v l_{4{ }_{2}} \mathrm{y}$ orden 27; véase el Cuadro 4); $\mathrm{y}$, en tercer lugar, los trabajadores con ingresos precarios extremos, con contrato precario y sin seguridad social (Subnivel ${ }_{27}$ y orden 15; véase el Cuadro 4).

En todos los casos, las condiciones de precariedad laboral extrema que más predominan en las combinaciones son: el ingreso precario extremo, el contrato precario, la jornada precaria y sin seguridad social. El hecho de que las probabilidades de ocurrencia se concentren en los mismos niveles y subniveles de precariedad laboral, así como en el mismo orden de relevancia a lo largo de los 15 años de referencia, señala la persistencia del fenómeno, que lejos de mostrar un desplazamiento hacia niveles menores de precariedad laboral extrema, se confirma como un elemento casi intrínseco del mercado laboral de México.

De acuerdo con las simulaciones de los modelos probit-pool para medir los efectos marginales en niveles y subniveles de precariedad laboral extrema, el resultado general que destaca es que el grupo de variables que influye con mayor magnitud en los cambios de las probabilidades de ocurrencia son las del perfil socioeconómico y de formación escolar. Del conjunto de variables socioeconómicas, por orden de importancia, destacan: si el trabajador está casado, si es jefe de hogar y si es hombre, y todo indica que la edad no es tan importante cuando se analizan los cambios en la probabilidad en los niveles y subniveles de precariedad laboral extrema (Cuadro 4). En cuanto al sentido de los efectos, destaca que los trabajadores casados y jefes de hogar influyen positivamente en la modificación de las probabilidades en grupos de precariedad extrema baja (Nivel 2 y Subnivel $_{26}$ ) y negativamente en los grupos de precariedad alta (Nivel 4, y Subniveles 2-7 y 4_2). En tanto, los trabajadores hombres tienen mayor posibilidad de reducir la probabilidad en los niveles y subniveles de precariedad extrema bajos y aumentarla en los niveles más altos. Aunque los trabajadores con mayor edad no son tan relevantes en los cambios de probabilidad, llama la atención que los efectos son negativos en los niveles y subniveles de precariedad extrema muy altos (véase el Nivel 4 y Subnivel 4_2 del Cuadro 4).

En cuanto a la influencia de los programas, en general no tiene relevancia; destaca que en los subniveles de precariedad extrema bajos (Subni- 
$v l_{26}$ ) fue positivo, y en los de mayor precariedad extrema cambió el signo a negativo (Subniveles 2_7).

Aunque los indicadores de capital humano son los menos importantes, es interesante cómo afectan en los cambios de las probabilidades de los niveles y subniveles de precariedad laboral extrema. La escolaridad es el factor más importante en valor numérico, seguido por la experiencia y la capacitación. Pero los cambios en la escolaridad y la experiencia tienden a aumentar la probabilidad en niveles y subniveles de precariedad bajos ( $\mathrm{Ni}$ vel 2 y Subnivel ${ }_{26}$ ) y reducirla en los más altos (Nivel 4, y Subniveles 2_7 y 4 2). Mientras que las horas de capacitación tienen una influencia contraria a la escolaridad y la experiencia, debido a que tienden a reducir las probabilidades en niveles y subniveles de precariedad extrema bajos y aumentarla en niveles y subniveles de precariedad más altos.

\section{Conclusiones}

Esta investigación se enfocó en el empleo precario extremo con ingresos por su trabajo iguales a la línea de pobreza extrema urbana, en condiciones laborales adversas que ponen a los trabajadores en una situación de riesgos por ser altamente reemplazables en el mercado laboral, por la falta de prestaciones a las que tienen derecho, sin seguridad social, con jornadas fuera de la Ley Federal del Trabajo, contratos temporales o ausencia de ellos y sin afiliación sindical. Con la metodología de grupos que propusimos se tiene la ventaja de identificar empleos heterogéneos en condiciones de precariedad extrema, no explícito en otros estudios (Oliveira, 2006; Castillo, 2009; Mora, 2005, 2012; Escotia, 2011; Román y Cervantes, 2013), con base en la combinación de condiciones laborales que definen niveles y subniveles de empleos con precariedad laboral extrema. La población ocupada con empleo en condiciones de precariedad extrema se estimó en 2.6 millones de trabajadores, equivalente al 5.2\% de la población ocupada total en 2018. Se ha caracterizado por su tendencia creciente en cantidad de trabajadores y estacionado entre 5 y $6 \%$ de la población ocupada total desde 2009, lo cual indica que es un fenómeno del mercado laboral mexicano que prácticamente no depende del ciclo económico y parece estar más vinculado con el desempleo. Este vínculo entre empleo precario y desempleo lo propuso García (2010), y nosotros lo confirmamos al encontrar que una de las condiciones laborales con mayor probabilidad de ocurrencia es la falta de contrato, que definimos en un sentido más amplio con respecto a otros estudios, como la población ocupada sin contrato escrito, y en el 
caso de tenerlo, si es temporal, por obra determinada o del tipo no especificado. El empleo precario sin contrato tiende a ser más vulnerable porque pone al trabajador en una posición de riesgo permanente ante el desempleo (Olsthoorm, 2014).

Con la metodología de grupos se construyeron niveles y subniveles para caracterizar la heterogeneidad del empleo en condiciones de precariedad extrema, pero los resultados mostraron un fenómeno altamente concentrado en la combinación entre cuatro condiciones laborales: ingresos precarios en la línea de pobreza extrema urbana; con contrato precario; jornada laboral precaria, que incluye a los trabajadores con menos de 34 horas y con la jornada extendida de más de 48 horas; y sin seguridad social, que es la falta de acceso a la atención médica de instituciones como: IMSS, Naval, Militar o PEMEX, ISSSTE, ISSSTE estatal y otras. Las combinaciones dos y cuatro de condiciones laborales representaron el 92\% en los niveles y $93 \%$ en los subniveles de precariedad laboral extrema. Las condiciones laborales con mayor probabilidad de ocurrencia fueron: 1) ingreso precario extremo, con contrato y jornada laboral precarios, con $41 \%$ de ocurrencia; 2) ingreso precario extremo, con contrato y jornada laboral precarios, sin seguridad social y sin sindicato, con $32 \%$ de ocurrencia; y 3 ) ingreso precario extremo, con contrato precario y sin seguridad, con $20 \%$ de ocurrencia.

Con los resultados de los modelos probit-pool y la simulación de los efectos marginales, se pudieron contrastar algunas hipótesis. En primer lugar, se concluyó que los niveles y subniveles de precariedad extrema más altos no se vinculan con los trabajadores que son hombres y jefes del hogar, lo cual implica un sesgo de género hacia las mujeres con empleos precarios extremos. Los trabajadores con edades mayores al promedio de 40 años y casados tienen mayor probabilidad de tener un empleo con mayor precariedad laboral extrema; y, dado que sólo el $25 \%$ de los empleados con precariedad extrema manifestó recibir apoyos de programas sociales, aunque los efectos encontrados en las simulaciones son consistentes, la conclusión es que no existe un vínculo claro entre los apoyos de programas sociales y la probabilidad de reducir los riesgos del empleo en las peores condiciones de precariedad laboral extrema.

También se pudo concluir que la probabilidad de localizar empleos con mayores niveles y subniveles de precariedad laboral extrema es más alta en zonas urbanas, pero se reduce cuando el trabajador pertenece a empresas pequeñas, medianas y grandes donde se producen bienes del sector minero, eléctrico, manufacturas y construcción; y, en contraste, la probabilidad aumenta cuando el trabajador se localiza en empresas micro en los sectores 
primario o servicios. Finalmente, se contrastó la hipótesis que establece que el mayor nivel educativo reduce la probabilidad de obtener un empleo con las peores condiciones de precariedad laboral extrema (Olsthoorm, 2014). Aunque se encontró que la escolaridad y la experiencia sí reducen la probabilidad en trabajadores con las peores condiciones laborales de empleo precario extremo. La magnitud de los efectos encontrados en la simulación de los cambios marginales nos llevó a concluir que la mayor preparación educativa (primaria) y experiencia de los trabajadores no son suficientes para reducir su condición de precariedad extrema.

\section{Bibliografía}

Agresti, A. (2002). Categorical data. University of Florida: Wiley-Interscience.

Barattini, M. (2009). El trabajo precario en la era de la globalización. ¿Es posible la organización? Revista de la Universidad Bolivariana, 8(24), 17-37. Recuperado de https://scielo.conicyt.cl/pdf/polis/v8n24/art02. pdf

Bayón, M. C. (2006). Precariedad social en México y Argentina: tendencias, expresiones y trayectorias nacionales. Revista de la CEPAL, 88, 133-152. Recuperado de https://repositorio.cepal.org/bitstream/hand le/11362/11107/088133152_es.pdf?sequence $=1$ yisAllowed=y

Bilbao, A. (1999). El empleo precario. Seguridad de la economía e inseguridad del trabajo. Madrid, España: CAES.

Bonilla, R. (2015). Informalidad y precariedad laboral en el Distrito Federal. La economía de sobrevivencia. Economía Informa, 391, 6984. Recuperado de https://www.sciencedirect.com/science/article/pii/ S0185084915000067

Cabrer, B., Sancho, P. y Serrano, D. (2001). Microeconometría y decisión. Madrid, España: Pirámide.

Cano, E. (1998). La lógica de la precariedad laboral: el caso de la industria valenciana del mueble. Cuadernos de Relaciones Laborales, 13, 207227. Recuperado de http://revistas.ucm.es/index.php/CRLA/article/ view/CRLA9898220207A/32523

Cano, E. (2004). Formas, percepciones y consecuencias de la precariedad. Mientras Tanto, 93, 67-81. Recuperado de https://www.jstor.org/ stable/27820778?seq=1\#metadata_info_tab_contents

Castel, R. (1997). La metamorfosis social de la cuestión social. Una crónica del salariado. España: Paidós Ibérica. 
Castillo, D. (2001). Los nuevos precarios, ¿mujeres u hombres? Tendencias en el mercado de trabajo urbano en Panamá, 1982-1999. Papales de Población, 27, 99-145. Recuperado de http://www.scielo.org.mx/scielo. php?script $=$ sci_arttextypid $=$ S1405-74252001000100006

Castillo, D. (2009). Los nuevos trabajadores precarios. México: Universidad Autónoma del Estado de México / Miguel Ángel Porrúa.

Escotia, A. (2011). Medición de la precariedad: otros tipos de relación entre las variables, s.l.: Ponencia para el VII Congreso de la Asociación Mexicana de Estudios del Trabajo.

Gallo, M. E. (2003). Precariedad laboral en el mercado de trabajo marplatense. Faces, 9(16), 109-131. Recuperado de http://nulan.mdp.edu. ar/110/1/FACES_n16_109-131.pdf

García, B. (2009). Los mercados de trabajo urbanos de México a principios del siglo XXI. Revista Mexicana de Sociología, 71(1), pp. 5-46. Recuperado de http://revistamexicanadesociologia.unam.mx/index.php/ $\mathrm{rms} /$ article/view/17742/16922

García, B. (2010). Precariedad laboral y desempleo en México, 2000-2009. Ponencia presentada en la X Reunión Nacional de la Sociedad Mexicana de Demografía, México.

García, B. (2012). La precarización laboral y el empleo en México (20002009). Ciudad de México: Plaza y Valdés.

García, B. y Oliveria, O. de. (2001). Transformaciones recientes en los mercados de trabajo metropolitanos de México: 1990-1998. Estudios Sociológicos, 19(3), 653-689. Recuperado de http://www.redalyc.org/ articulo.oa? $\mathrm{id}=59805705$

García Mora, M. B. (2004). Efectos de la educación sobre los determinantes de la satisfacción laboral en España. Un análisis de los beneficios monetarios y no monetarios en el mercado de trabajo mediante modelos logit ordenados. Tesis doctoral. Universidad de Valencia, España. Recuperado de https://www.tdx.cat/handle/10803/9694

Green, W. (2003). Econometric analysis. New Jersey: Prentice Hall.

Guadarrama, R., Hualde, A. y López, S. (2012). Precariedad laboral y heterogeneidad ocupacional: una propuesta teórico-metodológica. Revista Mexicana de Sociología, 74(2), 213-243. Recuperado de http://revistamexicanadesociologia.unam.mx/index.php/rms/article/ view/31199/28889

Kerns, G. (2011). Introduction to probability and statistics using $R$. s.l.: GNU, Free Documentation License. 
Mora, M. (2005). Ajuste y empleo: notas sobre la precarización del empleo asalariado. Revista de Ciencias Sociales, 2(108), 27-39. Recuperado de http://www.redalyc.org/articulo.oa?id=15310803

Mora, M. (2012). La medición de la precariedad laboral: problemas metodológicos y alternativas de solución. Revista Trabajo, 5(9), 89-124. Recuperado de https://minormora.colmex.mx/images/PDF/medici_ precar.pdf

Neffa, J. C. (2010). Naturaleza y significación del trabajo/empleo precario. Buenos Aires, Argentina: Miño y Dávila.

Neffa, J. C., Tupac, D. y Pérez, P. (2000). Actividad empleo y desempleo: conceptos y definiciones. Buenos Aires, Argentina: Trabajo y Sociedad.

OIT. (2012). Del trabajo precario al trabajo decente. Ginebra, Suiza: Organización Internacional del Trabajo.

Oliveira, O. de. (2006). Jóvenes y precariedad laboral en México. Papeles de Población, 12(49), 37-73. Recuperado de http://www.scielo.org.mx/ scielo.php?script=sci_arttextypid=S1405-74252006000300003

Olsthoorm, M. (2014). Measuring precarious employment: A proposal for two indicators of precarious employment based on set-theory and tested with dutch labor market-data. Social Indicators Research, 119(1), 421-441. Recuperado de https://link.springer.com/article/10.1007/ s11205-013-0480-y

Quiñones, C. y Rodríguez, S. (2015). La reforma laboral, la precarización del trabajo y el principio de estabilidad en el empleo. Revista Latinoamericana de Derecho Social, 21, 179-201. Recuperado de https://www. redalyc.org/pdf/4296/429640697008.pdf

Recio, A. (2015). Precariedad laboral: del neoliberalismo a la búsqueda de un modelo alternativo. Barcelona, España: Universidad Autónoma de Barcelona.

Rodríguez-Oreggia, E. y Silva, L. (2009). Construcción de un índice de condiciones laborales por estados para México. Gestión y Política Pública, 18(1), 149-178. Recuperado http://www.scielo.org.mx/scielo. php?script=sci_arttext\&pid=S1405-10792009000100005

Rojas, G. y Salas, C. (2008). La precarización del empleo en México, 19952004. Revista Latinoamericana de Estudios del Trabajo, 13(19), pp. 39-78. Recuperado de http://alast.info/relet_ojs/index.php/relet/article/ view/226

Román, Y. (2013). Impactos sociodemográficos y económicos en la precariedad laboral de los jóvenes en México. Región y Sociedad, 25(58), 165-202. Recuperado de http://www.scielo.org.mx/scielo.php?script $=$ sci_arttext\&pid $=$ S1870-39252013000300006 
Román, Y. y Cervantes, D. (2013). El empleo precario de jóvenes asalariados en México. El caso de Toluca, Tijuana y Mérida (2005-2010). Revista Facultad de Ciencias Económicos: Investigación y Reflexión, 21(1), 43-74. Recuperado de http://www.scielo.org.co/pdf/rfce/v21n1/ v21n1a05.pdf

Rubio, J. (2010). Precariedad laboral en México. Una propuesta de medición integral. Revista Enfoques: Ciencia Política y Administración Pública, 8(13), 77-87. Recuperado de http://www.redalyc.org/articulo. oa? id $=96016546006$

Ruiz, P. y Ordaz, J. L. (2011). Evolución reciente del empleo y desempleo en México. Economía UNAM, 8(23), 91-105. Recuperada de http:// www.revistas.unam.mx/index.php/ecu/article/view/44995/40549

Salas, C. (2000). Otra faceta de la dualidad económica: trabajo y empleo precario en el México actual. Revista Trabajo, 2(3), 119-133.

Shao, J. (2003). Mathematical statistics. Nueva York: Springer-Verlag.

Spanos, A. (2003). Probability theory and statistical inference. Econometric modeling with observational data. Cambridge: Cambridge University Press.

Weller, J. (2000). Reformas económicas, crecimiento y empleo: los mercados de trabajo en América Latina y el Caribe. Santiago de Chile: CEPAL.

Weller, J. (2017). Empleo en América Latina y el Caribe. Santiago de Chile: CEPAL, Naciones Unidas.

\section{Acerca de los autores}

Miguel Ángel Mendoza González es doctor en Economía por la Universidad Nacional Autónoma de México, institución donde labora como profesor-investigador de tiempo completo en el Posgrado de la Facultad de Economía. Pertenece al Sistema Nacional de Investigadores, nivel II. Es especialista en temas de crecimiento y desarrollo económico, capital humano, diferenciales y discriminación salarial por género, industria y clase creativa, y evaluación de política pública. Es tutor de maestría y doctorado, con experiencia docente en temas de modelación económica con técnicas estadísticas, matemáticas y econométricas, estadística matemática, análisis y econometría espacial, y sistemas de información geográfica. ORCID: http://orcid.org/0000-0001-7433-5194 
Entre sus más recientes publicaciones se encuentran:

Serván-Mori., E. y Chivardi, C., Mendoza-Gónzalez, M. A. y Nigenda, G. (2018). A longitudinal assessment of the technical efficiency in the outpatient production of maternal health services in Mexico. Health Policy and Planning, 33(8), 888-897. Recuperado de https://doi.org/10.1093/ heapol/czy074

Villagra-Piña, A., Mendoza-González, M. A. y Quintana-Romero, L. (2018). Un enfoque comparativo sobre la integración y apertura comercial en el crecimiento económico de la Unión Europea y América Latina. Revista Mexicana de Economía y Finanzas, 13(1), 27-52. Recuperado de http:// www.scielo.org.mx/pdf/rmef/v13n1/2448-6795-rmef-13-01-27.pdf

Mendoza-González, M. A., Cardero García, M. E. y Ortiz García, A. (2017). Algunos hechos estilizados y explicativos sobre el diferencial y la discriminación salarial por sexo en México, 1987-2015. Investigación Económica, 76(301), 103-135. Recuperado de http://www.revistas.unam. mx/index.php/rie/article/view/61969

Selene Fabiola Cruz Calderón es economista por la Universidad Autónoma del Estado de México (UAEM), maestra en Economía por la Universidad Nacional Autónoma de México (UNAM) y estudiante de doctorado en la Universidad Complutense de Madrid (UCM). Es especialista en temas de economía laboral y política social. Ha participado en la evaluación de programas sociales elaborados por la Coordinación de la Investigación Científica de la UNAM para diversas instituciones públicas. ORCID: http:/orcid. org/0000-0003-0119-6300

Marcos Valdivia López es doctor en Economía por la New School for Social Research de Nueva York. Es investigador de tiempo completo en el Centro Regional de Investigaciones Multidisciplinarias en el Programa de Estudios Regionales de la Universidad Nacional Autónoma de México, y también es tutor y profesor del Posgrado de Economía de dicha institución, en el campo de Economía Urbana y Regional. Es miembro del SNI. Ha publicado artículos académicos y libros en las áreas de la economía espacial y computacional, geografía económica, economía de la migración, mercados laborales y economía creativa, entre otros temas relacionados con la economía de las ciudades. ORCID: http://orcid.org/0000-0003-0119-6300

Entre sus publicaciones se encuentra: 
Valdivia, M. y Cuadrado-Roura, J. (coords). (2017). La economía de las actividades creativas: una perspectiva desde España y México. Cuernavaca, Morelos: Universidad Nacional Autónoma de México, Centro Regional de Investigaciones Multidisciplinarias; Alcalá de Henares, Madrid: Universidad de Alcalá.

Recepción: 21 de agosto de 2017.

Aceptación: 17 de octubre de 2018. 